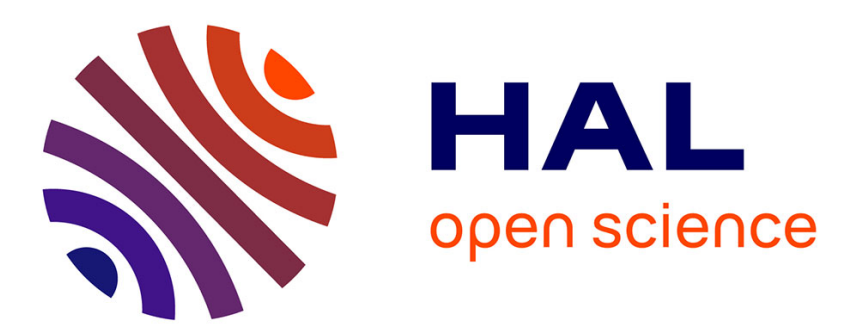

\title{
Vertex-based Compatible Discrete Operator schemes on polyhedral meshes for advection-diffusion equations
}

Pierre Cantin, Alexandre Ern

\section{To cite this version:}

Pierre Cantin, Alexandre Ern. Vertex-based Compatible Discrete Operator schemes on polyhedral meshes for advection-diffusion equations. Computational Methods in Applied Mathematics, 2016, 16 (2), pp.187-212. hal-01141290v3

\section{HAL Id: hal-01141290 \\ https://hal.science/hal-01141290v3}

Submitted on 18 Feb 2016

HAL is a multi-disciplinary open access archive for the deposit and dissemination of scientific research documents, whether they are published or not. The documents may come from teaching and research institutions in France or abroad, or from public or private research centers.
L'archive ouverte pluridisciplinaire HAL, est destinée au dépôt et à la diffusion de documents scientifiques de niveau recherche, publiés ou non, émanant des établissements d'enseignement et de recherche français ou étrangers, des laboratoires publics ou privés. 


\title{
Vertex-Based Compatible Discrete Operator Schemes on Polyhedral Meshes for Advection-Diffusion Equations
}

\author{
Pierre Cantin ${ }^{*, 1,2}$ and Alexandre Ern ${ }^{\dagger, 1}$ \\ ${ }^{1}$ Université Paris-Est, CERMICS (ENPC), 77455 Marne la Vallée Cedex 2, France \\ ${ }^{2} \mathrm{EDF}$ R\&D, 6 quai Watier, 78401 Chatou BP 49, France
}

\begin{abstract}
We devise and analyze vertex-based, Péclet-robust, lowest-order schemes for advection-diffusion equations that support polyhedral meshes. The schemes are formulated using Compatible Discrete Operators (CDO), namely primal and dual discrete differential operators, a discrete contraction operator for advection, and a discrete Hodge operator for diffusion. Moreover, discrete boundary operators are devised to weakly enforce Dirichlet boundary conditions. The analysis sheds new light on the theory of Friedrichs' operators at the purely algebraic level. Moreover, an extension of the stability analysis hinging on infsup conditions is presented to incorporate divergence-free velocity fields under some assumptions. Error bounds and convergence rates for smooth solutions are derived, and numerical results are presented on three-dimensional polyhedral meshes.
\end{abstract}

Keywords. Polyhedral meshes, compatible discretization, advection, diffusion, Peclet robustness, divergencefree velocity

AMS Subject Classification. 65N15, 65N12, 76Rxx

\section{Introduction}

The goal of this work is to approximate the scalar-valued function $p: \Omega \rightarrow \mathbb{R}$ solving the following advectiondiffusion problem:

$$
\begin{aligned}
-\nabla \cdot(\boldsymbol{\lambda} \nabla p)+\boldsymbol{\beta} \cdot \nabla p=s & \text { a.e. in } \Omega \\
p=p_{\mathrm{D}} & \text { a.e. on } \partial \Omega
\end{aligned}
$$

where $\Omega$ is a bounded polyhedral connected subset of $\mathbb{R}^{3}$ with boundary $\partial \Omega$ and outward unit normal $\boldsymbol{n}, \boldsymbol{\lambda}$ a bounded, symmetric, uniformly positive-definite tensor-valued field in $\Omega, \boldsymbol{\beta}$ a vector-valued field in $\boldsymbol{W}^{1, \infty}(\Omega), s \in L^{2}(\Omega)$, and $p_{\mathrm{D}} \in H^{t}(\partial \Omega), t>1$. We use boldface fonts for vector- and tensor-valued quantities. In addition to the classical assumption on the sign of $\nabla \cdot \boldsymbol{\beta}$, we also include in our analysis an extension to the case of divergence-free advection; see below. This extension is by no means straightforward and is rarely addressed in the literature. We also briefly discuss the (simpler) variants where the advection term is written in divergence form and where there is a zero-order reactive term. Of particular interest is the

*Email : pircantin@gmail.com

${ }^{\dagger}$ Email : alexandre.ern@enpc.fr 
robustness of the approximation with respect to the local Péclet number measuring the relative magnitude of advection and diffusion scaled by the local mesh size. Hence, we also study the pure advection problem with $\boldsymbol{\lambda}$ vanishing uniformly in (1.1a) and boundary condition (1.1b) modified so as to prescribe the Dirichlet condition only on the inflow part of $\partial \Omega$.

The goal of the present work is to devise and analyze a lowest-order, vertex-based scheme for the advection-diffusion problem (1.1) that is robust with respect to the Péclet number and that supports polyhedral meshes. The present scheme can be viewed as a polyhedral extension of Finite Element/Finite Volume (FE/FV) schemes which combine a finite element treatment of the diffusive term and an upwind finite volume treatment of the advection term. Such schemes were devised by Baba and Tabata [3] for triangular meshes using dual cells around vertices as control volumes and by Ohmori and Ushijima [33] using diamond cells around edges. Schemes of these type have been considered more recently by Angot et al. [1], Bochev et al. [6], Hilhorst and Vohralík [27], see also references therein.

A salient feature of the present work is that we investigate a possible way of relaxing the usual assumption on the advection velocity which in the present setting states that

( $\boldsymbol{\beta 1})$ There exists a real number $\tau>0$ such that $-\nabla \cdot \boldsymbol{\beta} \geq \tau^{-1}$ a.e. in $\Omega$.

This assumption, which is classically used to achieve $L^{2}$-stability by means of a coercivity argument, does not allow one to consider divergence-free advection velocities (a simple example could be a constant advection velocity). In the present work, we extend the analysis so as to cover the following situation:

( $\beta 2) ~ \nabla \cdot \boldsymbol{\beta}=0$, and there exist a real number $\tau>0$ and a function $\zeta \in W^{1, \infty}(\Omega)$, such that $\zeta \geq 1$ and $-\nabla \cdot(\zeta \boldsymbol{\beta}) \geq \tau^{-1}$ a.e. in $\Omega$.

Assumption ( $\boldsymbol{\beta 2}$ ) has been considered in Devinatz et al. [18] and more recently in Ayuso and Marini [2] for discontinuous Galerkin (dG) schemes and in Deuring et al. [17] for FE/FV schemes. Sufficient conditions on the existence of the function $\zeta$ can be found in [2]; loosely speaking, assumption $(\boldsymbol{\beta 2})$ is reasonable when the velocity field $\beta$ has no closed curves and no stationary point in $\Omega$. We also notice that the lower bound $\zeta \geq 1$ is not restrictive since the condition $-\nabla \cdot(\zeta \boldsymbol{\beta}) \geq \tau^{-1}$ is invariant by adding a constant to $\zeta$. Moreover, the function $\zeta$ is non-dimensional, and the real number $\tau$ in both $(\boldsymbol{\beta 1})$ and $(\boldsymbol{\beta 2})$ represents a reference time. The analysis with assumption $(\boldsymbol{\beta 2})$ is more complex than with assumption $(\boldsymbol{\beta 1})$ since stability now hinges on an inf-sup condition, and the handling of diffusive terms is delicate.

We formulate our schemes using the Compatible Discrete Operator (CDO) framework studied in [9] for diffusion problems and in [10] for the Stokes equations; see also Hiptmair for discrete Hodge operators [28, 29], Tarhasaari et al. [36] and Bossavit [11, 12]. The motivation for using the CDO formalism is twofold: we can hinge on previous work concerning diffusion, and the present schemes can serve as a starting point for CDO schemes discretizing the convective term in the Navier-Stokes equations. The algebraic viewpoint of CDO schemes also sheds new light on the theory of Friedrichs' operators [21, 22, 23] in the context of (discrete) contraction operators.

Our work contains two new contributions concerning CDO schemes. The first one is to devise a CDO scheme for pure advection. Here, the key idea is to build a discrete contraction (or interior product) operator that is the discrete counterpart of the map $\boldsymbol{g} \mapsto \boldsymbol{\beta} \cdot \boldsymbol{g}$. This way, the advective derivative $\boldsymbol{\beta} \cdot \nabla p$ can be discretized by two distinct operators: a (well-known) topological discrete gradient operator mapping degrees of freedom (DoFs) attached to vertices to DoFs attached to edges and the above discrete contraction operator. Our second new contribution is to devise a CDO scheme for diffusion with weakly enforced 
boundary conditions. Indeed, as for stabilized finite element and dG methods, weak enforcement of Dirichlet conditions yields better results for under-resolved outflow layers. To this purpose, we extend Nitsche's boundary penalty method [32] to the CDO setting.

Let us put our work in perspective with existing schemes. For pure advection, we emphasize that the CDO scheme is essentially an upwind finite volume scheme on a dual mesh with vertex-based control volumes. Thus, the analysis uses similar techniques to those used for dG methods; see Johnson and Pitkäranta [30], Brezzi et al. [14], and [20]. We also mention the following recent approaches to discretize pure advection equations in the setting of differential geometry. Using the notion of extrusion defined by Bossavit in [13], Heumann and Hiptmair [25] and Mullen et al. [31] proposed a discretization of interior products respectively on triangular and Cartesian meshes. Stabilized Galerkin methods for differential forms are considered by Heumann and Hiptmair [26]. Palha et al. [34] proposed another approach using the wedge product as the adjoint operator of the interior product. Furthermore, for advection-diffusion, the present CDO scheme is, to our knowledge, the first polyhedral discretization that is only vertex-based and that is robust for dominant advection up to the limit of zero diffusion. Another framework for vertex-based polyhedral schemes for elliptic PDEs is that of Virtual Element Methods (VEM), see Beirão da Veiga et al. [4]. The difference is that we use explicit reconstruction functions (typically piecewise constant on subcells) and we treat dominant advection, but our schemes are only of lowest-order. A first alternative to vertex-based schemes are face-based schemes: an arbitrary-order, Péclet-robust, face-based scheme for advection-diffusion has been recently analyzed by Di Pietro et al. [19] (see also Beirão da Veiga et al. [5] for the lowest-order and diffusion-dominated case). Another alternative is to use a cell-based dG method, but the treatment of diffusion requires introducing interior penalty parameters and using order $k \geq 1$ in cells leading to an increase of DoFs.

The material is organized as follows. In Section 2, we introduce the main notation for the discrete setting. In Section 3, we devise and analyze CDO schemes for pure advection. In Section 4, we treat advection-diffusion. Both sections 3 and 4 focus on assumption $(\boldsymbol{\beta 1})$ for the velocity field for simplicity. In Section 5 , we revisit the analysis in the case of a divergence-free velocity field under assumption $(\boldsymbol{\beta 2})$. In Section 6 , we present numerical results on three-dimensional polyhedral meshes. Finally, in Section 7 , we collect some proofs of technical results.

\section{Discrete setting}

In this section, we introduce the main ingredients underlying the discrete setting: mesh entities, degrees of freedom, and discrete differential operators. For brevity, we focus on the ideas needed in what follows; a broader presentation can be found in Bossavit [11, 12], Tonti [37] and, more recently, in Bonelle [7].

\subsection{Mesh entities}

The primal mesh of the three-dimensional domain $\Omega$ is denoted $\mathrm{M}:=\{\mathrm{V}, \mathrm{E}, \mathrm{F}, \mathrm{C}\}$, where $\mathrm{V}$ collects the mesh vertices generically denoted $v$ (0-cells), E collects edges denoted $e$ (1-cells), F collects faces denoted $f$ (2-cells), and $\mathrm{C}$ collects cells denoted $c$ (3-cells). The mesh $\mathrm{M}$ has the structure of a cellular complex in the sense that the boundary of a $k$-cell in $\mathrm{M}, 1 \leq k \leq 3$, can be decomposed into $(k-1)$-cells in $\mathrm{M}$, see Christiansen [15]. All the primal mesh entities are oriented; in what follows, we only need to assign a fixed orientation to any edge $e \in \mathrm{E}$ by means of a unit tangent vector $\boldsymbol{t}_{e}$. 
CDO schemes are formulated by considering a dual mesh $\widetilde{M}:=\{\widetilde{V}, \widetilde{E}, \widetilde{F}, \widetilde{C}\}$ such that there is a one-toone pairing between primal vertices and dual cells, primal edges and dual faces, and so on (see Figure 1). In particular, $\tilde{f}(e)$ denotes the dual face associated with the primal edge $e \in \mathrm{E}$, and $\tilde{c}(v)$ the dual cell associated with the primal vertex $v \in \mathrm{V}$. Dual mesh entities are oriented by the associated primal entity; for instance, $\boldsymbol{n}_{\tilde{f}(e)}$ is the unit normal vector to $\tilde{f}(e)$ oriented by $\boldsymbol{t}_{e}$. There are many possibilities to build a dual mesh. In this work, we assume that primal faces are planar and star-shaped w.r.t. their barycenter and that primal cells are star-shaped w.r.t. their barycenter, and we consider the fully barycentric dual mesh built using barycenters of all the primal mesh entities.

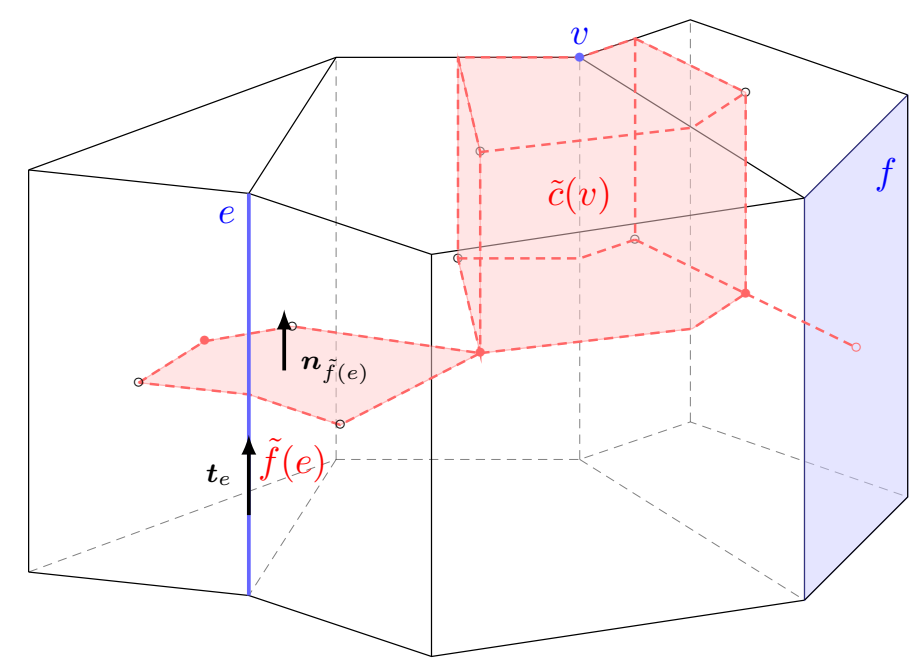

Figure 1: Illustration of primal and dual mesh entities

In what follows, we assume that the meshes $\mathrm{M}$ and $\widetilde{\mathrm{M}}$ satisfy a regularity requirement stating that there exists a common simplicial sub-complex of $\mathrm{M}$ and $\widetilde{M}$ (i.e., any $k$-simplex, $1 \leq k \leq 3$, in this sub-complex belongs to only one $k$-cell of $\mathrm{M}$ and of $\widetilde{\mathrm{M}}$ ) such that all the $k$-simplices are shape-regular in the usual sense of Ciarlet and any $k$-cell of $\mathrm{M}$ or $\widetilde{\mathrm{M}}$ contains a uniformly bounded number of $k$-simplices. This mesh regularity assumption is only needed to analyze the schemes, but not for implementation. For any primal or dual mesh entity $x, h_{x}$ denotes the diameter of $x$; moreover, when deriving convergence rates for smooth solutions, we use $h$ to denote the largest primal cell diameter. To alleviate the notation, we abbreviate $A \lesssim B$ the inequality $A \leq c B$ with positive constant $c$ whose value can change at each occurrence as long as it is uniform with respect to the mesh-size and the model parameters.

Since boundary conditions are enforced weakly in this work, we consider mesh entities at the boundary. The trace of the primal mesh $\mathrm{M}$ at the boundary $\partial \Omega$ defines a cellular complex $\mathrm{M}^{\partial}:=\left\{\mathrm{V}^{\partial}, \mathrm{E}^{\partial}, \mathrm{F}^{\partial}\right\}$, where $\mathrm{V}^{\partial}$ collects all the primal vertices lying at the boundary, and so on. Instead, the dual mesh has no entities lying at the boundary, so that we introduce an additional set of dual faces $\widetilde{\mathrm{F}}^{\partial}=\left\{\tilde{f}^{\partial}(v) \mid v \in \mathrm{V}^{\partial}\right\}$ with $\tilde{f}^{\partial}(v):=\partial \tilde{c}(v) \cap \partial \Omega$; observe the one-to-one pairing between $\mathrm{V}^{\partial}$ and $\widetilde{\mathrm{F}}^{\partial}$.

\subsection{Degrees of freedom}

The degrees of freedom (DoFs) of discrete fields are attached to mesh entities according to their physical nature. For instance, the degrees of freedom of a discrete potential field (0-cochain) are attached to vertices, either primal or dual ones. In this work, we focus on vertex-based CDO schemes where these DoFs are attached to primal vertices. For a discrete potential $\mathrm{q}$, we use the notation $\mathrm{q} \in \mathcal{V} \equiv \mathbb{R}^{\#(\mathrm{~V})}$, where $\mathcal{V}$ is the 
vector space composed of DoFs attached to primal vertices and \#(V) denotes the cardinality of the set $\mathrm{V}$. We write $\mathrm{q}_{v}$ for the value of $\mathrm{q}$ attached to the vertex $v \in \mathrm{V}$. We also consider discrete circulation fields (1cochains) in $\mathcal{E}$ (attached to primal edges), discrete flux fields (2-cochains) in $\widetilde{\mathcal{F}}$ (attached to dual faces), and discrete density fields (3-cochains) in $\widetilde{\mathcal{C}}$ (attached to dual cells). Owing to the one-to-one pairing between primal and dual mesh entities, the vector spaces $\mathcal{V}$ and $\widetilde{\mathcal{C}}$ are isomorphic, as well as the vector spaces $\mathcal{E}$ and $\widetilde{\mathcal{F}}$. This leads us to define the algebraic duality products $\llbracket \mathrm{q}, \mathrm{s} \rrbracket_{\mathcal{V} \tilde{\mathcal{C}}}=\sum_{v \in \mathrm{V}} \mathrm{q}_{v} \mathrm{~s}_{\tilde{\mathcal{C}}(v)}$ for all $(\mathrm{q}, \mathrm{s}) \in \mathcal{V} \times \widetilde{\mathcal{C}}$, and $\llbracket \mathrm{g}, \phi \rrbracket_{\mathcal{E} \tilde{\mathcal{F}}}=\sum_{e \in \mathrm{E}} \mathrm{g}_{e} \phi_{\tilde{f}(e)}$ for all $(\mathrm{g}, \phi) \in \mathcal{E} \times \widetilde{\mathcal{F}}$.

To weakly enforce boundary conditions, we consider discrete fields at the boundary, and with obvious notation, we introduce the isomorphic vector spaces $\mathcal{V}^{\partial}$ and $\tilde{\mathcal{F}}^{\partial}$, along with the algebraic duality product $\llbracket \mathbf{q}^{\partial}, \phi^{\partial} \rrbracket_{\left(\mathcal{V} \tilde{F}^{\partial}\right.}=\sum_{v \in \mathrm{V}^{\partial}} \mathbf{q}_{v}^{\partial} \phi_{\tilde{f}^{\partial}(v)}^{\partial}$. Furthermore, for all $\mathbf{q} \in \mathcal{V}$, we use the notation $\mathbf{q}^{\partial} \in \mathcal{V}^{\partial}$ with $\mathbf{q}_{v}^{\partial}=\mathbf{q}_{v}$ for all $v \in \mathrm{V}^{\partial}$, i.e., $\mathrm{q}^{\partial}$ collects the DoFs of $\mathrm{q}$ attached to boundary vertices.

To measure the approximation error resulting from CDO schemes and to discretize the source terms and boundary conditions, we define DoFs for continuous fields. One possibility is to consider the classical de Rham maps (we also consider other choices below) for smooth enough fields. In what follows, we consider the maps $\mathrm{R}_{\mathcal{V}}: S_{\mathcal{V}}(\Omega) \rightarrow \mathcal{V}, \mathrm{R}_{\mathcal{E}}: \boldsymbol{S}_{\mathcal{E}}(\Omega) \rightarrow \mathcal{E}, \mathrm{R}_{\widetilde{\mathcal{F}}}: \boldsymbol{S}_{\widetilde{\mathcal{F}}}(\Omega) \rightarrow \widetilde{\mathcal{F}}$ and $\mathrm{R}_{\widetilde{\mathcal{C}}}: S_{\widetilde{\mathcal{C}}}(\Omega) \rightarrow \widetilde{\mathcal{C}}$ such that

$$
\begin{aligned}
& \left(\mathrm{R}_{\mathcal{V}}(p)\right)_{v}=p(v) \quad \text { for all } v \in \mathrm{V}, \quad\left(\mathrm{R}_{\mathcal{E}}(\boldsymbol{g})\right)_{e}=\int_{e} \boldsymbol{t}_{e} \cdot \boldsymbol{g} d e \quad \text { for all } e \in \mathrm{E}, \\
& \left(\mathrm{R}_{\widetilde{\mathcal{F}}}(\phi)\right)_{\tilde{f}}=\int_{\tilde{f}} \boldsymbol{n}_{\tilde{f}} \cdot \phi d \tilde{f} \quad \text { for all } \tilde{f} \in \widetilde{\mathrm{F}}, \quad\left(\mathrm{R}_{\widetilde{\mathcal{C}}}(s)\right)_{\tilde{c}}=\int_{\tilde{c}} s d \tilde{c} \quad \text { for all } \tilde{c} \in \widetilde{\mathrm{C}} .
\end{aligned}
$$

Possible choices for the domains of the de Rham maps are $S_{\mathcal{V}}(\Omega)=H^{s}(\Omega)$ with $s>\frac{3}{2}, \boldsymbol{S}_{\mathcal{E}}(\Omega)=\boldsymbol{H}^{s}(\Omega)$ with $s>1$ or $\boldsymbol{S}_{\mathcal{E}}(\Omega)=\left\{\boldsymbol{g} \in \boldsymbol{L}^{p}(\Omega), \nabla \times \boldsymbol{g} \in \boldsymbol{L}^{t}(\Omega)\right\}$ with $p>2$ and $t>\frac{1}{2}$, and $\boldsymbol{S}_{\widetilde{\mathcal{F}}}(\Omega)=\boldsymbol{H}^{s}(\Omega)$ with $s>\frac{1}{2}$ or $\boldsymbol{S}_{\widetilde{\mathcal{F}}}(\Omega)=\left\{\boldsymbol{\phi} \in \boldsymbol{L}^{p}(\Omega), \nabla \cdot \boldsymbol{\phi} \in L^{2}(\Omega)\right\}$ with $p>2$ and $S_{\widetilde{\mathcal{C}}}=L^{1}(\Omega)$. At the boundary, we use the maps $\mathrm{R}_{\mathcal{V}^{\partial}}: S_{\mathcal{V}^{\partial}}(\partial \Omega) \rightarrow \mathcal{V}^{\partial}$ and $\mathrm{R}_{\widetilde{\mathcal{F}}^{\partial}}: S_{\widetilde{\mathcal{F}}^{\partial}}(\partial \Omega) \rightarrow \widetilde{\mathcal{F}}^{\partial}$ such that

$$
\left(\mathrm{R}_{\mathcal{V}^{\partial}}(p)\right)_{v}=p(v) \text { for all } v \in \mathrm{V}^{\partial}, \quad\left(\mathrm{R}_{\widetilde{\mathcal{F}}^{\partial}}(\phi)\right)_{\tilde{f}^{\partial}}=\int_{\tilde{f}^{\partial}} \phi d \tilde{f} \text { for all } \tilde{f}^{\partial} \in \widetilde{\mathrm{F}}^{\partial},
$$

with $S_{\mathcal{V}^{\partial}}(\partial \Omega)=H^{t}(\partial \Omega), t>1$, and $S_{\widetilde{\mathcal{F}}^{\partial}}(\partial \Omega)=L^{1}(\partial \Omega)$.

\subsection{Discrete differential operators}

For all $v \in \mathrm{V}$ and all $e \in \mathrm{E}$, we set $\iota_{v, e}=1$ if $v$ is the extremity of $e$ toward which $\boldsymbol{t}_{e}$ points, $\iota_{v, e}=-1$ if $v$ is the other extremity of $e$, and $\iota_{v, e}=0$ if $v$ is not an extremity of $e$. The discrete gradient operator GRAD $: \mathcal{V} \rightarrow \mathcal{E}$ is defined such that $(\operatorname{GRAD}(\mathbf{q}))_{e}=\sum_{v \in \mathrm{V}} \iota_{v, e} \mathbf{q}_{v}$ for all $\mathbf{q} \in \mathcal{V}$; note that the algebraic representation of GRAD is a rectangular matrix with entries in $\{0, \pm 1\}$. We also define a discrete dual divergence operator $\widetilde{\operatorname{DIV}}: \widetilde{\mathcal{F}} \rightarrow \widetilde{\mathcal{C}}$ such that $(\widetilde{\operatorname{DIV}}(\phi))_{\tilde{c}(v)}=\sum_{\tilde{f}(e) \in \widetilde{\mathcal{F}}} \iota_{\tilde{f}(e), \tilde{c}(v)} \phi_{\tilde{f}(e)}$ for all $\phi \in \widetilde{\mathcal{F}}$, with $\iota_{\tilde{f}(e), \tilde{c}(v)}=-\iota_{v, e}$. Observe that $\iota_{\tilde{f}(e), \tilde{c}(v)}=1$ (resp., -1) if $\tilde{f}(e)$ is a face of the dual cell $\tilde{c}(v)$ such that $\boldsymbol{n}_{\tilde{f}(e)}$ points outward (resp., inward) $\tilde{c}(v)$, and $\iota_{\tilde{f}(e), \tilde{c}(v)}=0$ if $\tilde{f}(e)$ is not a face of $\tilde{c}(v)$.

The following discrete adjunction property holds:

$$
\llbracket \operatorname{GRAD}(\mathrm{q}), \phi \rrbracket_{\mathcal{E} \tilde{\mathcal{F}}}=-\llbracket \mathrm{q}, \widetilde{\operatorname{DIV}}(\phi) \rrbracket_{\mathcal{V} \tilde{\mathcal{c}}}, \quad \forall(\mathbf{q}, \phi) \in \mathcal{V} \times \widetilde{\mathcal{F}}
$$


Other important properties are the two following commuting properties with the de Rham maps:

$$
\begin{array}{rlrl}
\operatorname{GRAD}\left(\mathrm{R}_{\mathcal{V}}(p)\right)=\mathrm{R}_{\mathcal{E}}(\nabla p), & & \forall p \in S_{\mathcal{V}}(\Omega), \\
\llbracket \mathbf{q}, \mathrm{R}_{\widetilde{\mathcal{C}}}(\nabla \cdot \boldsymbol{\phi}) \rrbracket_{\mathcal{V} \tilde{\mathcal{C}}}=\llbracket \mathbf{q}, \widetilde{\operatorname{DIV}}\left(\mathrm{R}_{\widetilde{\mathcal{F}}}(\boldsymbol{\phi})\right) \rrbracket_{\mathcal{V} \tilde{\mathcal{C}}}+\llbracket \mathbf{q}^{\partial}, \mathrm{R}_{\widetilde{\mathcal{F}} \partial}(\boldsymbol{n} \cdot \boldsymbol{\phi}) \rrbracket_{(\mathcal{\mathcal { F }})^{\partial}}, & \forall \boldsymbol{\phi} \in \boldsymbol{S}_{\widetilde{\mathcal{F}}}(\Omega), \quad \forall \mathbf{q} \in \mathcal{V} .
\end{array}
$$

Remark 2.1 (Boundary term). We have chosen the above definition of DIV since it is naturally associated with the dual mesh where dual cells attached to boundary vertices are, by definition, not closed by dual faces. An alternative choice is to modify the definition of DIV to include dual boundary faces attached to primal boundary vertices; by doing so, the boundary term appears in (2.1) and no longer in (2.2b).

\subsection{Restriction to primal cells and boundary faces}

It is convenient to localize discrete objects to a primal cell or to a boundary face. Let $c \in \mathrm{C}$. We define the local subsets $\mathrm{V}_{c}:=\{v \in \mathrm{V} \mid v \in \partial c\}$ (collecting the vertices of the cell $c$ ) and $\mathrm{E}_{c}:=\{e \in \mathrm{E} \mid e \subset \partial c\}$ (collecting the edges of the cell $c$ ). For all $e \in \mathrm{E}_{c}$, we define $\tilde{f}_{c}(e):=\tilde{f}(e) \cap c$ as the portion of the dual face $\tilde{f}(e)$ inside $c$ (see Figure 2, left panel), and we set $\widetilde{\mathrm{F}}_{c}:=\left\{\tilde{f}_{c}(e) ; e \in \mathrm{E}_{c}\right\}$. The vector space $\mathcal{E}_{c}$ is composed of the DoFs of discrete circulation fields $\mathrm{g} \in \mathcal{E}$ attached to $\mathrm{E}_{c}$; similarly for $\mathcal{V}_{c}$ and for $\widetilde{\mathcal{F}}_{c}$. The de Rham maps $\mathrm{R}_{\mathcal{E}_{c}}$ and $\mathrm{R}_{\widetilde{\mathcal{F}}_{c}}$ are such that $\left(\mathrm{R}_{\mathcal{E}_{c}}(\boldsymbol{g})\right)_{e}=\int_{e}\left(\boldsymbol{t}_{e} \cdot \boldsymbol{g}\right) d e$ for all $e \in \mathrm{E}_{c}$, and $\left(\mathrm{R}_{\widetilde{\mathcal{F}}_{c}}(\boldsymbol{\phi})\right)_{\tilde{f}}=\int_{\tilde{f}}\left(\boldsymbol{n}_{\tilde{f}} \cdot \boldsymbol{\phi}\right) d \tilde{f}$ for all $\tilde{f} \in \widetilde{\mathrm{F}}_{c}$. The local discrete gradient operator $\operatorname{GRAD}_{c}: \mathcal{V}_{c} \rightarrow \mathcal{E}_{c}$ is defined similarly to GRAD. We also define the following local norms:

$$
\|\mathrm{q}\|_{2, \mathcal{V}_{c}}^{2}:=h_{c}^{3} \sum_{v \in \mathrm{V}_{c}} \mathrm{q}_{v}^{2}, \quad\|\mathrm{~g}\|_{2, \mathcal{E}_{c}}^{2}:=h_{c} \sum_{e \in \mathrm{E}_{c}} \mathrm{~g}_{e}^{2},
$$

for all $\mathrm{q} \in \mathcal{V}_{c}$ and all $\mathrm{g} \in \mathcal{E}_{c}$. The global counterparts of these norms are assembled cell-wise as $\|\mathrm{q}\|_{2, \mathcal{V}}^{2}:=$ $\sum_{c \in \mathrm{C}}\|\mathrm{q}\|_{2, \mathcal{V}_{c}}^{2}$ and $\|\mathrm{g}\|_{2, \mathcal{E}}^{2}:=\sum_{c \in \mathrm{C}}\|\mathrm{g}\|_{2, \mathcal{E}_{c}}^{2}$, for all $\mathrm{q} \in \mathcal{V}$ and all $\mathrm{g} \in \mathcal{E}$.

Let now $f \in \mathrm{F}^{\partial}$ be a primal boundary face. We define the local subset $\mathrm{V}_{f}^{\partial}=\left\{v \in \mathrm{V}^{\partial} \mid v \in \partial f\right\}$, collecting boundary vertices attached to $f$. For all $v \in \mathrm{V}_{f}^{\partial}$, we define $\tilde{f}_{f}^{\partial}(v):=\tilde{f}^{\partial}(v) \cap f$ as the portion of the dual face $\tilde{f}^{\partial}(v)$ inside $f$ (see Figure 2, right panel), and we set $\widetilde{\mathrm{F}}_{f}^{\partial}:=\left\{\tilde{f}_{f}^{\partial}(v) ; v \in \mathrm{V}_{f}^{\partial}\right\}$. The vector space $\mathcal{V}_{f}^{\partial}$ is composed of the DoFs of discrete boundary potential fields $\mathrm{q}^{\partial}$ attached to $\mathrm{V}_{f}^{\partial}$; similarly for $\widetilde{\mathcal{F}}_{f}^{\partial}$. The de Rham map $\mathrm{R}_{\widetilde{\mathcal{F}}_{f}^{\partial}}$ is such that $\left(\mathrm{R}_{\widetilde{\mathcal{F}}_{f}^{\partial}}(\phi)\right)_{\tilde{f}^{\partial}}=\int_{\tilde{f}^{\partial}} \phi d \tilde{f}^{\partial}$ for all $\tilde{f}^{\partial} \in \widetilde{\mathrm{F}}_{f}^{\partial}$.
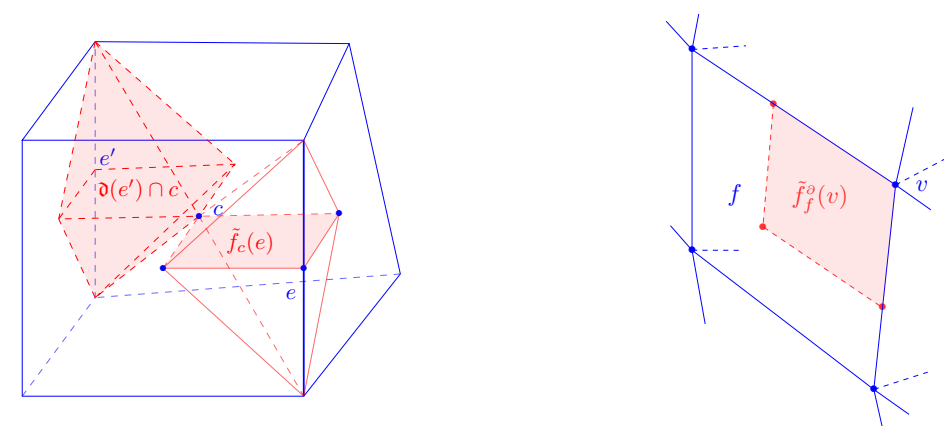

Figure 2: Illustration of local mesh entities for a cell (left) and a boundary face (right) 


\section{Pure advection}

This section is concerned with the derivation and analysis of vertex-based CDO schemes for the pure advection problem

$$
\begin{aligned}
\boldsymbol{\beta} \cdot \nabla p=s & \text { a.e. in } \Omega, \\
p=p_{\mathrm{D}} & \text { a.e. on } \partial \Omega^{-},
\end{aligned}
$$

where $\boldsymbol{\beta}$ satisfies assumption $(\boldsymbol{\beta 1})$, and $\partial \Omega^{ \pm}:=\{x \in \partial \Omega \mid \pm \boldsymbol{\beta} \cdot \boldsymbol{n}(x)>0\}$ correspond to the inflow $\left(\partial \Omega^{-}\right)$ and outflow $\left(\partial \Omega^{+}\right)$parts of the boundary $(\beta$ can be tangential to some part of the boundary). In what follows, we consider the positive and negative parts of $\boldsymbol{\beta} \cdot \boldsymbol{n}$ defined as $(\boldsymbol{\beta} \cdot \boldsymbol{n})^{ \pm}=\frac{1}{2}(|\boldsymbol{\beta} \cdot \boldsymbol{n}| \pm \boldsymbol{\beta} \cdot \boldsymbol{n}) \geq 0$. We introduce the graph space $V_{\boldsymbol{\beta}}(\Omega)=\left\{q \in L^{2}(\Omega) ; \boldsymbol{\beta} \cdot \nabla q \in L^{2}(\Omega)\right\}$; functions in the graph space have a trace in $L^{2}(|\boldsymbol{\beta} \cdot \boldsymbol{n}| ; \partial \Omega)$ provided $\partial \Omega^{-}$and $\partial \Omega^{+}$are well separated (see [21]). In this context, a well-posed weak formulation of problem (3.1) (see $[21,20]$ ) is as follows: Find $p \in V_{\boldsymbol{\beta}}(\Omega)$ such that

$$
a_{\beta}(p, q)=\int_{\Omega} q s d \Omega+\int_{\partial \Omega} q \phi_{\mathrm{D}} d \partial \Omega
$$

for all $q \in V_{\boldsymbol{\beta}}(\Omega)$, with boundary flux $\phi_{\mathrm{D}}=(\boldsymbol{\beta} \cdot \boldsymbol{n})^{-} p_{\mathrm{D}}$, and with bilinear form

$$
a_{\boldsymbol{\beta}}(p, q)=\int_{\Omega} q(\boldsymbol{\beta} \cdot \nabla p) d \Omega+\int_{\partial \Omega} q(\boldsymbol{\beta} \cdot \boldsymbol{n})^{-} p d \partial \Omega .
$$

Note that the boundary integrals vanish outside $\partial \Omega^{-}$.

\subsection{CDO scheme}

Vertex-based CDO schemes for pure advection are built using two discrete operators: a discrete contraction operator $\left.\right|_{\beta} ^{\mathcal{E}}: \mathcal{E} \rightarrow \widetilde{\mathcal{C}}$, which is the discrete counterpart of the map $\boldsymbol{g} \mapsto \boldsymbol{\beta} \cdot \boldsymbol{g}$, and a discrete boundary Hodge operator $\mathrm{H}_{\alpha}^{\partial}: \mathcal{V}^{\partial} \rightarrow \widetilde{\mathcal{F}}^{\partial}$ (indexed by a surface function $\alpha \in L^{\infty}(\partial \Omega)$ ) which is the discrete counterpart of the map $p \mapsto \alpha p$ at the boundary. Using these operators, the following discrete problem can be formulated: Find $\mathrm{p} \in \mathcal{V}$ such that

$$
\mathrm{A}_{\beta}(\mathrm{p}, \mathrm{q})=\llbracket \mathrm{q}, \mathrm{s} \rrbracket_{\mathcal{\mathcal { C }}}+\llbracket \mathrm{q}^{\partial}, \phi_{\mathrm{D}} \rrbracket_{(\mathcal{V} \tilde{\mathcal{F}})}, \quad \forall \mathrm{q} \in \mathcal{V}
$$

with bilinear form such that

$$
\mathrm{A}_{\boldsymbol{\beta}}(\mathrm{p}, \mathrm{q}):=\llbracket \mathrm{q}, \mathrm{I}_{\boldsymbol{\beta}}^{\mathcal{E} \widetilde{\mathcal{C}}}(\operatorname{GRAD}(\mathrm{p})) \rrbracket_{\mathcal{V} \tilde{\mathcal{C}}}+\llbracket \mathrm{q}^{\partial}, \mathrm{H}_{(\boldsymbol{\beta} \cdot \boldsymbol{n})^{-}}^{\partial}\left(\mathrm{p}^{\partial}\right) \rrbracket_{(\mathcal{V} \tilde{\mathcal{F}})^{\partial}}
$$

and where we have set $\mathrm{s}:=\mathrm{R}_{\widetilde{\mathcal{C}}}(s)$ and $\phi_{\mathrm{D}}:=\mathrm{R}_{\widetilde{\mathcal{F}}^{\partial}}\left(\phi_{\mathrm{D}}\right)$. A synthetic presentation of the scheme (3.4) is the so-called Tonti diagram shown in Figure 3.

In the spirit of Friedrichs operators [21,23], we assume that there is a second discrete contraction operator $I_{\beta}^{\mathcal{F}}$, which is the discrete counterpart of the map $p \mapsto \boldsymbol{\beta} p$, and such that the following two properties hold:

(I1) [Discrete Leibniz rule] The bilinear map on $\mathcal{V} \times \mathcal{V}$ such that

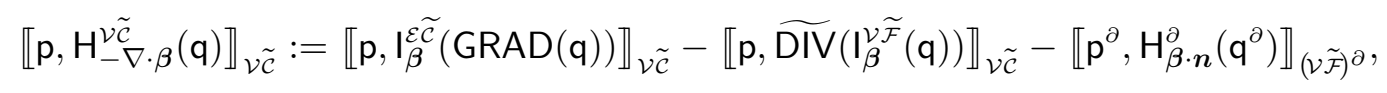

is symmetric and satisfies $\llbracket \mathrm{p}, \mathrm{H}_{-\nabla \cdot \boldsymbol{\beta}}^{\tilde{\nu}}(\mathrm{q}) \rrbracket_{\mathcal{V} \tilde{\mathcal{C}}} \geq\left(\operatorname{ess}_{\inf }-\nabla \cdot \boldsymbol{\beta}\right)\|\mathrm{q}\|_{2, \mathcal{V}}^{2}$ for all $\mathrm{q} \in \mathcal{V}$, so that $\mathrm{H}_{-\nabla \cdot \boldsymbol{\beta}}^{\tilde{\mathcal{C}}}$ is monotone under assumption $(\boldsymbol{\beta 1})$; see Remark 3.1 below for the boundary term in (3.6). 


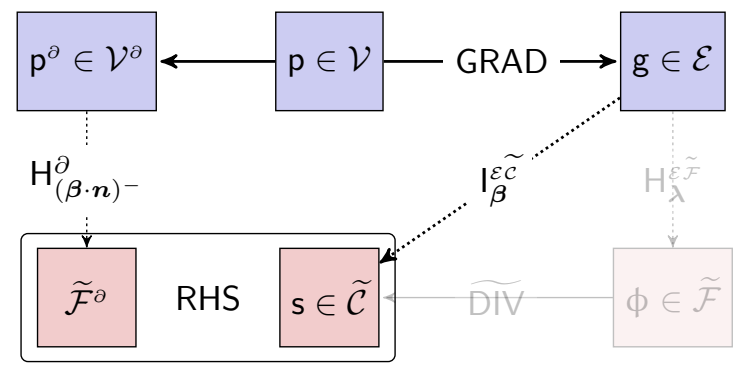

Figure 3: Tonti diagram of the vertex-based CDO scheme for pure advection

(I2) [Discrete integration by parts] The bilinear map on $\mathcal{V} \times \mathcal{V}$ such that

$$
\langle\mathrm{p}, \mathrm{q}\rangle_{\mathrm{upw}, \beta}:=\llbracket \mathrm{p}, \mathrm{I}_{\beta}^{\mathcal{E} \widetilde{\mathcal{C}}}(\mathrm{GRAD}(\mathrm{q})) \rrbracket_{\mathcal{\nu} \tilde{\mathcal{C}}}+\llbracket \mathrm{q}, \widetilde{\operatorname{DIV}}\left(\mathrm{I}_{\beta}^{\mathcal{F}}(\mathrm{p})\right) \rrbracket_{\mathcal{V} \tilde{\mathcal{C}}}
$$

defines a semi-inner product.

Concerning the discrete boundary Hodge operator, we assume the following:

$\left(\mathbf{H}^{\partial}\right)$ For all $\alpha \in L^{\infty}(\partial \Omega), \mathbf{H}_{\alpha}^{\partial}$ is self-adjoint, and it depends linearly and monotonically on $\alpha$ (i.e., $\alpha \geq \alpha^{\prime}$ a.e. in $\partial \Omega$ implies that $\mathrm{H}_{\alpha}^{\partial} \geq \mathrm{H}_{\alpha^{\prime}}^{\partial}$ in the sense of quadratic forms), so that whenever $\alpha \geq 0, \llbracket \mathrm{q}^{\partial}, \mathrm{H}_{\alpha}^{\partial}\left(\mathrm{p}^{\partial}\right) \rrbracket_{(\nu \tilde{\mathcal{F}})}{ }^{\partial}$ defines a semi-inner product on $\mathcal{V}^{\partial} \times \mathcal{V}^{\partial}$.

Remark 3.1 (Discrete contraction operator). Recalling from Remark 2.1 that the discrete dual divergence operator DIV does not involve faces on the boundary $\partial \Omega$, property (I1) is the discrete counterpart of the Leibniz formula $-\int_{\Omega} p(\nabla \cdot \boldsymbol{\beta}) q d \Omega=\int_{\Omega} p(\boldsymbol{\beta} \cdot \nabla q) d \Omega-\int_{\Omega} p \nabla \cdot(\boldsymbol{\beta} q) d \Omega$, where the two rightmost terms in (3.6) form together the discrete counterpart of $\int_{\Omega} p \nabla \cdot(\boldsymbol{\beta} q) d \Omega$. Furthermore, property (I2) is the discrete counterpart of $\int_{\Omega} p(\boldsymbol{\beta} \cdot \nabla q) d \Omega+\int_{\Omega} q \nabla \cdot(\boldsymbol{\beta} p) d \Omega-\int_{\partial \Omega} q(\boldsymbol{\beta} \cdot \boldsymbol{n}) p d \partial \Omega=0$. At the discrete level, this quantity can be non-zero owing to the use of stabilization. We also notice that the symmetry of the map $\langle\mathrm{p}, \mathrm{q}\rangle_{\mathrm{upw}, \boldsymbol{\beta}}$ results from $\langle\mathrm{p}, \mathrm{q}\rangle_{\text {upw }, \beta}-\langle\mathrm{q}, \mathrm{p}\rangle_{\text {upw }, \beta}=\llbracket \mathrm{p}, \mathrm{H}_{-\nabla \cdot \beta}^{\tilde{\mathcal{C}}}(\mathrm{q}) \rrbracket_{\mathcal{\nu} \tilde{\mathcal{C}}}-\llbracket \mathrm{q}, \mathrm{H}_{-\nabla \cdot \beta}^{\tilde{\mathcal{C}}}(\mathrm{p}) \rrbracket_{\mathcal{\mathcal { C }}}=0$ where we have used the selfadjointness of $\mathrm{H}_{\beta \cdot \boldsymbol{n}}^{\partial}$ and of $\mathrm{H}_{-\nabla \cdot \boldsymbol{\beta}}^{\mathcal{\nu}}$. Finally, we observe that $\mathbf{I}_{\beta}^{\mathcal{E}} \widetilde{\mathcal{C}}$ does not, in general, depend linearly on its argument $\beta$ owing to the use of stabilization.

Remark 3.2 (Conservative advection). A possible variant of (3.1) is to consider the conservative form of the advective derivative. The PDE becomes $\nabla \cdot(\boldsymbol{\beta} p)=s$ in $\Omega$, and a Dirichlet boundary condition can still be enforced at the inflow boundary. Assumption $(\boldsymbol{\beta 1})$ is then modified as follows: There exists a real number $\tau>0$ such that $\nabla \cdot \boldsymbol{\beta} \geq \tau^{-1}$ a.e. in $\Omega$. The discrete bilinear form then becomes

$$
\mathrm{A}_{\boldsymbol{\beta}}(\mathrm{p}, \mathrm{q})=\llbracket \mathrm{q}, \widetilde{\operatorname{DIV}}\left(\mathrm{I}_{\boldsymbol{\beta}}^{\mathcal{\mathcal { F }}}(\mathrm{p})\right) \rrbracket_{\mathcal{\nu} \tilde{\mathcal{C}}}+\llbracket \mathrm{q}^{\partial}, \mathrm{H}_{(\boldsymbol{\beta} \cdot \boldsymbol{n})^{+}}^{\partial}\left(\mathrm{p}^{\partial}\right) \rrbracket_{(\mathcal{N} \tilde{\mathcal{F}})^{\partial}}
$$

The design of the discrete contraction and boundary Hodge operators still hinges on $(\mathbf{I 1})-(\mathbf{I} 2)$ and $\left(\mathbf{H}^{\partial}\right)$.

Remark 3.3 (Reaction). Another possible variant is to include a zero-order reaction term in the PDE which becomes $\boldsymbol{\beta} \cdot \nabla p+\mu p=s$ in $\Omega$ with Lipschitz reaction coefficient $\mu$ (the conservative form of the advective derivative can also be considered). Then, the reaction-related bilinear form $\mathrm{A}_{\mu}(\mathrm{p}, \mathrm{q})=\sum_{v \in \mathrm{V}} \mu_{v} \mathrm{p}_{v} \mathrm{q}_{v}$ is added to the discrete problem, where $\mu_{v}$ denotes (for instance) the mean-value of $\mu$ in $\tilde{c}(v)$. 


\subsection{Example: CDO scheme with upwinding}

Let us give a concrete example for the CDO scheme (3.4). We introduce the notation

$$
\beta_{e}:=\left(\mathrm{R}_{\widetilde{\mathcal{F}}}(\boldsymbol{\beta})\right)_{\tilde{f}(e)}=\int_{\tilde{f}(e)} \boldsymbol{\beta} \cdot \boldsymbol{n}_{\tilde{f}(e)} d \tilde{f}, \quad \forall e \in \mathrm{E} .
$$

We also set $\mathrm{E}_{v}:=\{e \in \mathrm{E} \mid v \in e\}$ for all $v \in \mathrm{V}$, and $\mathrm{V}_{e}:=\{v \in \mathrm{V} \mid v \in e\}$ for all $e \in \mathrm{E}$, and we use the notation $\boldsymbol{n}_{\tilde{f}(e), \tilde{c}(v)}=\iota_{\tilde{f}(e), \tilde{c}(v)} \boldsymbol{n}_{\tilde{f}(e)}$ for the unit normal to $\tilde{f}(e)$ pointing outward $\tilde{c}(v)$. For all $e \in \mathrm{E}$ and all $v \in \mathrm{V}_{e}$, we fix a real number $\Lambda_{v e} \in[-1,1]$ (the algebraic upwinding parameter) such that the following holds: For all $e \in \mathrm{E}$,

$(\Lambda 1) \sum_{v \in \mathrm{V}_{e}} \Lambda_{v e}=0$, and setting $\Lambda_{e}:=\frac{1}{2} \sum_{v \in \mathrm{V}_{e}} \iota_{\tilde{f}(e), \tilde{c}(v)} \Lambda_{v e}, \beta_{e} \Lambda_{e} \geq 0$ holds.

$(\Lambda 2)$ There exists $c_{\Lambda}>0$, uniform with respect to the mesh and the model parameters, such that $\beta_{e} \Lambda_{e} \geq$ $c_{\Lambda}\left|\beta_{e}\right|$.

The reason to distinguish the properties $\beta_{e} \Lambda_{e} \geq 0$ in $(\Lambda 1)$ and $\beta_{e} \Lambda_{e} \geq c_{\Lambda}\left|\beta_{e}\right|$ in $(\Lambda 2)$ is that the former is satisfied by the so-called centered scheme corresponding to $\Lambda_{v e}=0$ for all $v \in \mathrm{V}_{e}$, and the latter by an upwind scheme. Classical upwinding corresponds to the choice $\Lambda_{v e}=\operatorname{sign}\left(\iota_{\tilde{f}(e), \tilde{c}(v)} \beta_{e}\right)$ (with sign function $\operatorname{sign}(t)=-1$ if $t \in \mathbb{R}_{<0}, \operatorname{sign}(0)=0$, and $\operatorname{sign}(t)=1$ if $\left.t \in \mathbb{R}_{>0}\right)$, so that $(\Lambda 2)$ holds with $c_{\Lambda}=1$. With this choice, the solution delivered by the CDO scheme coincides with that of the upwind FV scheme on the dual mesh.

The discrete contraction operator $\left.\right|_{\beta} ^{\mathcal{E} \widetilde{\mathcal{C}}}: \mathcal{E} \rightarrow \widetilde{\mathcal{C}}$ is defined such that, for all $\mathrm{g} \in \mathcal{E}$,

$$
\left(\mathrm{I}_{\boldsymbol{\beta}}^{\mathcal{\varepsilon} \widetilde{C}}(\mathrm{~g})\right)_{\tilde{c}(v)}:=\sum_{e \in \mathrm{E}_{v}} \mathrm{~g}_{e} \frac{1}{2}\left(1-\Lambda_{v e}\right) \beta_{e}, \quad \forall v \in \mathrm{V}
$$

while the companion operator $\left.\right|_{\beta} ^{\mathcal{F}}: \mathcal{V} \rightarrow \widetilde{\mathcal{F}}$ is defined such that, for all $\mathrm{q} \in \mathcal{V}$,

$$
\left(\mathrm{I}_{\boldsymbol{\beta}}^{\nu \widetilde{\mathcal{F}}}(\mathbf{q})\right)_{\tilde{f}(e)}:=\sum_{v \in \mathrm{V}_{e}} \mathbf{q}_{v} \frac{1}{2}\left(1+\Lambda_{v e}\right) \beta_{e}, \quad \forall e \in \mathrm{E}
$$

Moreover, the discrete boundary Hodge operator $\mathrm{H}_{\alpha}^{\partial}: \mathcal{V}^{\partial} \rightarrow \tilde{\mathcal{F}}^{\partial}$ with $\alpha \in L^{\infty}(\partial \Omega)$ is defined such that, for all $\mathrm{q}^{2} \in \mathcal{V}^{2}$

$$
\left(\mathrm{H}_{\alpha}^{\partial}\left(\mathrm{q}^{\partial}\right)\right)_{\tilde{f}^{\partial}(v)}:=\mathrm{q}_{v}^{\partial} \int_{\tilde{f}^{\partial}(v)} \alpha d \tilde{f}, \quad \forall v \in \mathrm{V}^{\partial} .
$$

Observe that $\mathrm{H}_{\alpha}^{\partial}$ is algebraically represented by a diagonal matrix.

Remark 3.4 (Upwinding design). There are several possible variations in the geometric quantities considered for upwinding. Instead of considering the full dual face $\tilde{f}(e)$ as in (3.9), one possibility is to consider the average of the normal advection velocity on the dual sub-faces $\tilde{f}_{c}(e)$, and to design the upwinding parameters based on the sign of these quantities. In general, the smaller the underlying geometric objects, the larger the dissipation introduced by upwinding. The advantage of considering the dual sub-faces $\tilde{f}_{c}(e)$ is that upwinding is then compatible with the assembly of the scheme on primal cells.

Lemma 3.1 (Stability, (I1)-(I2)). Let the discrete contraction and surface Hodge operators be given by (3.10)- 


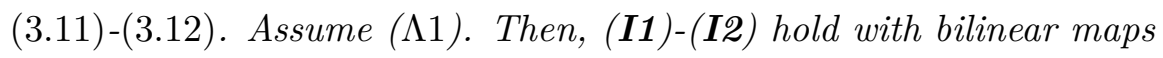

$$
\begin{aligned}
\llbracket \mathrm{p}, \mathrm{H}_{-\nabla \cdot \boldsymbol{\beta}}^{\nu \tilde{\mathcal{c}}}(\mathrm{q}) \rrbracket_{\mathcal{\nu} \tilde{\mathcal{c}}} & =\sum_{v \in \mathrm{V}} \mathrm{p}_{v} \mathrm{q}_{v} \int_{\tilde{c}(v)}-\nabla \cdot \boldsymbol{\beta} d \tilde{c} \\
\langle\mathrm{p}, \mathrm{q}\rangle_{\mathrm{upw}, \boldsymbol{\beta}} & =\sum_{e \in \mathrm{E}} \llbracket \mathrm{p} \rrbracket_{e} \llbracket \mathrm{q} \rrbracket_{e} \Lambda_{e} \beta_{e}
\end{aligned}
$$

so that $\mathrm{H}_{-\nabla \cdot \boldsymbol{\beta}}^{\nu \tilde{\mathcal{C}}}$ is algebraically represented by a diagonal matrix, and where $\llbracket \mathrm{q} \rrbracket e=\sum_{v \in \mathrm{V}_{e}} \iota_{\tilde{f}(e), \tilde{c}(v)} \mathrm{q}_{v}$ is the jump of q across the dual face $\tilde{f}(e)$ for all $\mathrm{q} \in \mathcal{V}$.

Remark 3.5 (First-order diffusion). Since $\llbracket \mathbf{q} \rrbracket_{e}=-(\operatorname{GRAD}(\mathbf{q}))_{e}$, the right-hand side of $(3.13 \mathrm{~b})$ corresponds to the jump penalty term considered in $\mathrm{dG}$ methods; for $k=0$, it can be interpreted as adding a first-order viscosity term.

Proof. Proof of (3.13a). Let $v \in \mathrm{V}$ and let $\mathrm{p}, \mathrm{q} \in \mathcal{V}$. Using (3.10)-(3.11), we infer that

$$
\begin{aligned}
& \llbracket \mathrm{p},\left.\right|_{\beta} ^{\mathcal{E} \widetilde{\mathcal{C}}}(\mathrm{GRAD}(\mathrm{q})) \rrbracket_{\mathcal{V} \tilde{\mathcal{C}}}=\sum_{v \in \mathrm{V}} \sum_{e \in \mathrm{E}_{v}} \sum_{v^{\prime} \in \mathrm{V}_{e}} \mathrm{p}_{v} \mathrm{q}_{v^{\prime}} \iota_{v^{\prime}, e} \frac{1}{2}\left(1-\Lambda_{v e}\right) \beta_{e}, \\
& \llbracket \mathrm{p}, \widetilde{\operatorname{DIV}}\left(\mathrm{I}_{\boldsymbol{\beta}}^{\mathcal{\mathcal { F }}}(\mathrm{q})\right) \rrbracket_{\mathcal{V} \tilde{\mathcal{C}}}=\sum_{v \in \mathrm{V}} \sum_{e \in \mathrm{E}_{v}} \sum_{v^{\prime} \in \mathrm{V}_{e}} \mathrm{p}_{v} \mathrm{q}_{v^{\prime}} \tilde{f}_{\tilde{f}(e), \tilde{c}(v)} \frac{1}{2}\left(1+\Lambda_{v^{\prime} e}\right) \beta_{e} .
\end{aligned}
$$

Using $\iota_{v, e}=-\iota_{\tilde{f}(e), \tilde{c}(v)}$, the definition of $\beta_{e}$, and $(\Lambda 1)$ leads to

$$
\llbracket \mathrm{p}, \mathrm{I}_{\boldsymbol{\beta}}^{\mathcal{\varepsilon} \widetilde{\mathcal{C}}}(\mathrm{GRAD}(\mathrm{q})) \rrbracket_{\mathcal{\mathcal { V }} \tilde{\mathcal{C}}}-\llbracket \mathrm{p}, \widetilde{\operatorname{DIV}}\left(\mathrm{I}_{\boldsymbol{\beta}}^{\mathcal{F}}(\mathrm{q})\right) \rrbracket_{\mathcal{V} \tilde{\mathcal{C}}}=\sum_{v \in \mathrm{V}} \mathrm{p}_{v} \mathrm{q}_{v} \sum_{e \in \mathrm{E}_{v}}-\int_{\tilde{f}(e)} \boldsymbol{\beta} \cdot \boldsymbol{n}_{\tilde{f}(e), \tilde{c}(v)} d \tilde{f}
$$

To conclude, we observe that if $v \in \mathrm{V} \backslash \mathrm{V}^{\partial}, \sum_{e \in \mathrm{E}_{v}} \int_{\tilde{f}(e)} \boldsymbol{\beta} \cdot \boldsymbol{n}_{\tilde{f}(e), \tilde{c}(v)} d \tilde{f}=\int_{\partial \tilde{c}(v)} \boldsymbol{\beta} \cdot \boldsymbol{n}_{\tilde{c}(v)} d \partial \tilde{c}=\int_{\tilde{c}(v)} \nabla \cdot \boldsymbol{\beta} d \tilde{c}$ owing to the divergence theorem, while for the boundary vertices, we use the definition (3.12) of the discrete boundary Hodge operator to infer that

$$
\sum_{v \in \mathrm{V}^{\partial}} \mathrm{p}_{v} \mathrm{q}_{v} \sum_{e \in \mathrm{E}_{v}} \int_{\tilde{f}(e)} \boldsymbol{\beta} \cdot \boldsymbol{n}_{\tilde{f}(e), \tilde{c}(v)} d \tilde{f}=\sum_{v \in \mathrm{V}^{\partial}} \mathrm{p}_{v} \mathrm{q}_{v} \int_{\tilde{c}(v)} \nabla \cdot \boldsymbol{\beta} d \tilde{c}-\llbracket \mathrm{p}^{\partial}, \mathrm{H}_{\boldsymbol{\beta} \cdot \boldsymbol{n}}^{\partial}\left(\mathrm{q}^{\partial}\right) \rrbracket_{(\nu \tilde{\mathcal{F}})^{\partial}} \cdot
$$

Proof of (3.13b). Using (3.10)-(3.11), the definition of $\llbracket \cdot \rrbracket_{e}$, and the adjunction property between GRAD and $\widetilde{\mathrm{DIV}}$, we infer that

$$
\begin{aligned}
\llbracket \mathrm{p}, \mathrm{I}_{\boldsymbol{\beta}}^{\mathcal{E} \widetilde{\mathcal{C}}}(\mathrm{GRAD}(\mathrm{q})) \rrbracket_{\mathcal{V} \tilde{\mathcal{C}}} & =\sum_{v \in \mathrm{V}} \sum_{e \in \mathrm{E}_{v}} \mathrm{p}_{v} \llbracket \mathrm{q} \rrbracket_{e} \frac{1}{2}\left(\Lambda_{v e}-1\right) \beta_{e}, \\
\llbracket \mathrm{q}, \widetilde{\operatorname{DIV}}\left(\mathrm{I}_{\boldsymbol{\beta}}^{\mathcal{\mathcal { F }}}(\mathrm{p})\right) \rrbracket_{\mathcal{V} \tilde{\mathcal{C}}} & =\sum_{e \in \mathrm{E}} \sum_{v \in \mathrm{V}_{e}} \llbracket \mathrm{q} \rrbracket_{e} \mathrm{p}_{v} \frac{1}{2}\left(1+\Lambda_{v e}\right) \beta_{e}
\end{aligned}
$$

Exchanging the summations in the first line leads to

$$
\llbracket \mathrm{p},\left.\right|_{\boldsymbol{\beta}} ^{\mathcal{E} \widetilde{\mathcal{C}}}(\operatorname{GRAD}(\mathrm{q})) \rrbracket_{\mathcal{V} \tilde{\mathcal{C}}}+\llbracket \mathrm{q}, \widetilde{\operatorname{DIV}}\left(\mathrm{I}_{\boldsymbol{\beta}}^{\mathcal{F}}(\mathrm{p})\right) \rrbracket_{\mathcal{V} \tilde{\mathcal{C}}}=\sum_{e \in \mathrm{E}} \sum_{v \in \mathrm{V}_{e}} \llbracket \mathrm{q} \rrbracket_{e} \mathrm{p}_{v} \Lambda_{v e} \beta_{e}
$$

Since $\sum_{v \in \mathrm{V}_{e}} \mathrm{p}_{v} \Lambda_{v e}=\llbracket \mathrm{p} \rrbracket_{e} \Lambda_{e}$ owing to $(\Lambda 1)$, we infer (3.13b). 


\subsection{Analysis: coercivity, consistency, and error bound}

We define the following stability norm for all $\mathrm{q} \in \mathcal{V}$ :

$$
\|\mathbf{q}\|_{a, \mathcal{V}}^{2}:=\tau^{-1}\|\mathbf{q}\|_{2, \mathcal{V}}^{2}+\|\mathbf{q}\|_{\text {upw }, \boldsymbol{\beta}}^{2}+\|\mathbf{q}\|_{|\boldsymbol{\beta} \cdot \boldsymbol{n}|}^{2},
$$

where $\tau>0$ results from assumption $(\boldsymbol{\beta 1}),\|\cdot\|_{2, \mathcal{V}}$ is defined in Section 2.4, and we can define $\|\mathrm{q}\|_{\text {upw, },}^{2}:=$ $\langle\mathbf{q}, \mathbf{q}\rangle_{\text {upw }, \boldsymbol{\beta}}$ from assumption $(\mathbf{I} 2)$, and $\|\mathbf{q}\|_{|\boldsymbol{\beta} \cdot \boldsymbol{n}|}^{2}:=\llbracket \mathbf{q}^{\partial}, \mathbf{H}_{|\boldsymbol{\beta} \cdot \boldsymbol{n}|}^{\partial}\left(\mathbf{q}^{\partial}\right) \rrbracket_{(\mathcal{V} \tilde{F})}$ from assumption $\left(\mathbf{H}^{\partial}\right)$.

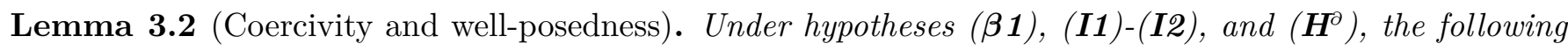
holds:

$$
\varrho\|\mathrm{q}\|_{a, \mathcal{V}}^{2} \leq \mathrm{A}_{\beta}(\mathrm{q}, \mathrm{q}), \quad \forall \mathrm{q} \in \mathcal{V}
$$

with $\varrho=\frac{1}{2}$. Consequently, (3.4) is well-posed.

Proof. Let $\mathrm{q} \in \mathcal{V}$. Since $(\mathbf{I} 1)-(\mathbf{I} 2)$ imply that

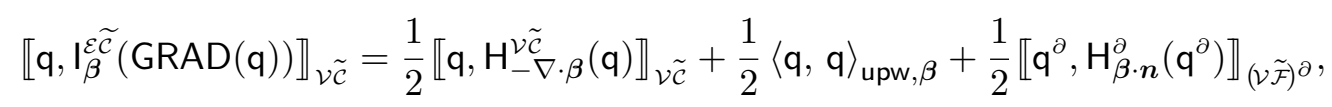

we infer that the quantity $A_{\beta}(q, q)$ can be rewritten as

$$
\mathrm{A}_{\boldsymbol{\beta}}(\mathrm{q}, \mathrm{q})=\frac{1}{2} \llbracket \mathrm{q}, \mathrm{H}_{-\nabla \cdot \boldsymbol{\nu}}^{\nu \tilde{\mathcal{C}}}(\mathrm{q}) \rrbracket_{\mathcal{\mathcal { C }}}+\frac{1}{2}\langle\mathrm{q}, \mathrm{q}\rangle_{\mathrm{upw}, \boldsymbol{\beta}}+\frac{1}{2} \llbracket \mathrm{q}^{\partial}, \mathrm{H}_{\boldsymbol{\beta} \cdot \boldsymbol{n}}^{\partial}\left(\mathrm{q}^{\partial}\right) \rrbracket_{(\nu \tilde{\mathcal{F}})^{\partial}}+\llbracket \mathrm{q}^{\partial}, \mathrm{H}_{(\boldsymbol{\beta} \cdot \boldsymbol{n})^{-}}\left(\mathrm{q}^{\partial}\right) \rrbracket_{(\mathcal{\nu} \tilde{\mathcal{F}})^{\partial}} .
$$

Owing to $\left(\mathbf{H}^{\partial}\right)$, the last two terms on the right-hand side can be recombined to yield

$$
\mathrm{A}_{\boldsymbol{\beta}}(\mathrm{q}, \mathrm{q})=\frac{1}{2} \llbracket \mathrm{q}, \mathrm{H}_{-\nabla \cdot \boldsymbol{\beta}}^{\mathcal{\nu}}(\mathrm{q}) \rrbracket_{\mathcal{V} \tilde{\mathcal{C}}}+\frac{1}{2}\langle\mathrm{q}, \mathrm{q}\rangle_{\mathrm{upw}, \boldsymbol{\beta}}+\frac{1}{2}\|\mathrm{q}\|_{|\boldsymbol{\beta} \cdot \boldsymbol{n}|}^{2}
$$

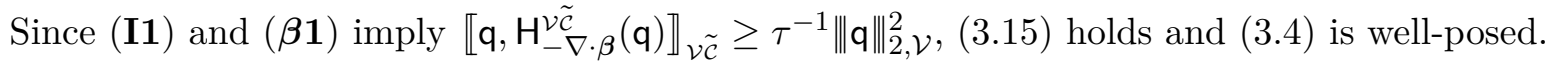

We now turn to the consistency of the CDO scheme (3.4) using commutators in the spirit of Bossavit [12], Hiptmair [28], and [9]. To write the consistency error, we consider the reduction map $\widehat{R}_{\mathcal{V}}: L^{1}(\Omega) \rightarrow \mathcal{V}$ such that $\left(\widehat{R}_{\mathcal{V}}(p)\right)_{v}$ equals the mean-value of $p$ in the dual cell $\tilde{c}(v)$, and the following three commutators:

$$
\begin{aligned}
\left\lfloor\mathrm{I}_{\boldsymbol{\beta}}^{\mathcal{\mathcal { F }}}, \widehat{\mathrm{R}}\right\rceil(q) & :=\mathrm{R}_{\widetilde{\mathcal{F}}}(\boldsymbol{\beta} q)-\mathrm{I}_{\boldsymbol{\beta}}^{\nu \widetilde{\mathcal{F}}}\left(\widehat{\mathrm{R}}_{\mathcal{V}}(q)\right), \\
\left\lfloor\mathrm{H}_{-\nabla \cdot \boldsymbol{\beta}}^{\nu \tilde{\mathcal{C}}}, \widehat{\mathrm{R}}\right\rceil(q) & :=\mathrm{R}_{\widetilde{\mathcal{C}}}((-\nabla \cdot \boldsymbol{\beta}) q)-\mathrm{H}_{-\nabla \cdot \boldsymbol{\beta}}^{\nu \tilde{\mathcal{C}}}\left(\widehat{\mathrm{R}}_{\mathcal{V}}(q)\right), \\
\left\lfloor\mathrm{H}_{(\boldsymbol{\beta} \cdot \boldsymbol{n})^{+}}^{\partial}, \widehat{\mathrm{R}}\right\rceil(q) & :=\mathrm{R}_{\widetilde{\mathcal{F}}^{\partial}}\left((\boldsymbol{\beta} \cdot \boldsymbol{n})^{+} q\right)-\mathrm{H}_{(\boldsymbol{\beta} \cdot \boldsymbol{n})^{+}}^{\partial}\left(\left(\widehat{\mathrm{R}}_{\mathcal{V}}(q)\right)^{\partial}\right),
\end{aligned}
$$

for all $q \in H^{s}(\Omega), s>\frac{1}{2}$, so that $q$ is in the domain of the maps $\mathrm{R}_{\widetilde{\mathcal{F}}}$ and $\mathrm{R}_{\widetilde{\mathcal{F}}}$.

Lemma 3.3 (Error bound). Let $p \in V_{\boldsymbol{\beta}}(\Omega)$ be the unique solution of (3.1) and let $\mathrm{p}$ be the unique solution of (3.4). Assume $p \in H^{s}(\Omega), s>\frac{1}{2}$. Then, under the assumptions of Lemma 3.2, the following holds:

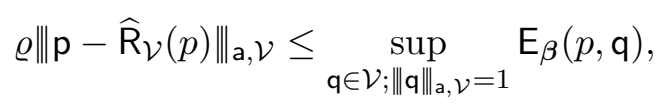

with consistency error defined as follows:

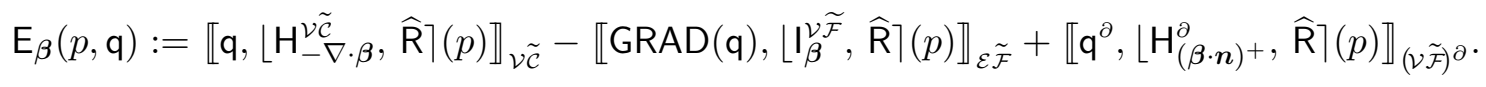


Proof. Owing to Lemma 3.2, it suffices to show that $\mathrm{A}_{\boldsymbol{\beta}}\left(\mathrm{p}-\widehat{\mathrm{R}}_{\mathcal{V}}(p), \mathrm{q}\right)=\mathrm{E}_{\boldsymbol{\beta}}(p, \mathrm{q})$. In the context of Friedrichs' systems, the derivation of the error bound hinges on integration by parts. In the CDO framework, we use the continuous and discrete Leibniz formulas, as well as the properties of the discrete differential operators. We observe that

$$
\begin{aligned}
& \mathrm{A}_{\boldsymbol{\beta}}(\mathrm{p}, \mathrm{q})=\llbracket \mathrm{q}, \mathrm{R}_{\widetilde{\mathcal{C}}}(\boldsymbol{\beta} \cdot \nabla p) \rrbracket_{\mathcal{V} \tilde{\mathcal{C}}}+\llbracket \mathrm{q}^{\partial}, \mathrm{R}_{\widetilde{\mathcal{F}}^{\partial}}\left((\boldsymbol{\beta} \cdot \boldsymbol{n})^{-} p\right) \rrbracket_{(\mathcal{\nu} \tilde{\mathcal{F}})^{\partial}} \\
& =\llbracket \mathbf{q}, \mathrm{R}_{\widetilde{\mathcal{C}}}(\nabla \cdot(\boldsymbol{\beta} p)) \rrbracket_{\mathcal{V} \tilde{\mathcal{C}}}+\llbracket \mathrm{q}, \mathrm{R}_{\widetilde{\mathcal{C}}}(-(\nabla \cdot \boldsymbol{\beta}) p) \rrbracket_{\mathcal{V} \tilde{\mathcal{C}}}+\llbracket \mathrm{q}^{\partial}, \mathrm{R}_{\widetilde{\mathcal{F}}^{\partial}}\left((\boldsymbol{\beta} \cdot \boldsymbol{n})^{-} p\right) \rrbracket_{(\mathcal{V} \tilde{\mathcal{F}})} \\
& =\llbracket \mathbf{q}, \widetilde{\operatorname{DIV}}\left(\mathrm{R}_{\widetilde{\mathcal{F}}}(\boldsymbol{\beta} p)\right) \rrbracket_{\mathcal{V} \tilde{\mathcal{C}}}+\llbracket \mathrm{q}, \mathrm{R}_{\widetilde{\mathcal{C}}}(-(\nabla \cdot \boldsymbol{\beta}) p) \rrbracket_{\mathcal{V} \tilde{\mathcal{C}}}+\llbracket \mathrm{q}^{\partial}, \mathrm{R}_{\widetilde{\mathcal{F}}}\left((\boldsymbol{\beta} \cdot \boldsymbol{n})^{+} p\right) \rrbracket_{(\nu \tilde{\mathcal{F}})^{\partial}} \\
& =-\llbracket \operatorname{GRAD}(\mathbf{q}), \mathrm{R}_{\widetilde{\mathcal{F}}}(\boldsymbol{\beta} p) \rrbracket_{\mathcal{E} \tilde{\mathcal{F}}}+\llbracket \mathbf{q}, \mathrm{R}_{\widetilde{\mathcal{C}}}(-(\nabla \cdot \boldsymbol{\beta}) p) \rrbracket_{\mathcal{V} \tilde{\mathcal{C}}}+\llbracket \mathrm{q}^{\partial}, \mathrm{R}_{\widetilde{\mathcal{F}}^{\partial}}\left((\boldsymbol{\beta} \cdot \boldsymbol{n})^{+} p\right) \rrbracket_{(\mathcal{V} \tilde{\mathcal{F}})},
\end{aligned}
$$

where we have used the continuous Leibniz formula (recall that $p$ is in the graph space), the discrete commuting property $(2.2 \mathrm{~b})$, the fact that $\boldsymbol{\beta} \cdot \boldsymbol{n}=(\boldsymbol{\beta} \cdot \boldsymbol{n})^{+}-(\boldsymbol{\beta} \cdot \boldsymbol{n})^{-}$, and the discrete adjunction property $(2.1)$. Moreover, setting $\widehat{p}=\widehat{R}_{\mathcal{V}}(p)$, we observe that

$$
\begin{aligned}
& \mathrm{A}_{\boldsymbol{\beta}}(\widehat{\mathrm{p}}, \mathrm{q})=\llbracket \mathrm{q}, \mathrm{l}_{\boldsymbol{\beta}}^{\mathcal{\mathcal { C }}}(\operatorname{GRAD}(\widehat{\mathrm{p}})) \rrbracket_{\mathcal{V} \tilde{\mathcal{C}}}+\llbracket \mathrm{q}^{\partial}, \mathrm{H}_{(\boldsymbol{\beta} \cdot \boldsymbol{n})^{-}}\left(\widehat{\mathrm{p}}^{\partial}\right) \rrbracket_{(\nu \tilde{\mathcal{F}})^{\partial}} \\
& =\llbracket \mathbf{q}, \widetilde{\operatorname{DIV}}\left(\mathrm{I}_{\boldsymbol{\beta}}^{\mathcal{\mathcal { F }}}(\widehat{\mathrm{p}})\right) \rrbracket_{\mathcal{V} \tilde{\mathcal{C}}}+\llbracket \mathbf{q}, \mathrm{H}_{-\nabla \cdot \boldsymbol{\beta}}^{\mathcal{\nu} \tilde{\mathrm{p}})} \rrbracket_{\mathcal{V} \tilde{\mathcal{C}}}+\llbracket \mathbf{q}^{\partial}, \mathrm{H}_{(\boldsymbol{\beta} \cdot \boldsymbol{n})^{+}}\left(\widehat{\mathrm{p}}^{\partial}\right) \rrbracket_{(\nu \tilde{\mathcal{F}})^{\partial}} \\
& =-\llbracket \operatorname{GRAD}(\mathbf{q}), \mathrm{I}_{\beta}^{\nu \widetilde{\mathcal{F}}}(\widehat{\mathbf{p}}) \rrbracket_{\mathcal{E} \tilde{\mathcal{F}}}+\llbracket \mathbf{q}, \mathrm{H}_{-\nabla \cdot \boldsymbol{\beta}}^{\nu \tilde{\mathcal{C}}}(\widehat{\mathbf{p}}) \rrbracket_{\mathcal{\nu} \tilde{\mathcal{C}}}+\llbracket \mathrm{q}^{\partial}, \mathrm{H}_{(\boldsymbol{\beta} \cdot \boldsymbol{n})}^{\partial}\left(\widehat{\mathbf{p}}^{\partial}\right) \rrbracket_{(\nu \tilde{\mathcal{F}})},
\end{aligned}
$$

where we have used the discrete Leibniz formula, assumption $\left(\mathbf{H}^{\partial}\right)$ (linearity) together with $\boldsymbol{\beta} \cdot \boldsymbol{n}=(\boldsymbol{\beta} \cdot \boldsymbol{n})^{+}-$ $(\boldsymbol{\beta} \cdot \boldsymbol{n})^{-}$, and the discrete adjunction property (2.1). The conclusion is straightforward.

Theorem 3.4 (Convergence rate). Assume ( $\boldsymbol{\beta} 1)$. Let $L_{\beta}$ be the Lipschitz constant of $\boldsymbol{\beta}$ and assume that $L_{\beta} \lesssim \tau^{-1}$. Let the discrete contraction and surface Hodge operators be given by (3.10)-(3.11)-(3.12). Assume $(\Lambda 1)-(\Lambda 2)$. Let $p$ be the unique solution of (3.1) and let $\mathrm{p}$ be the unique solution of (3.4). Assume that $p \in H^{1}(\Omega)$. Then, the following holds:

$$
\varrho\left\|\mathrm{p}-\widehat{\mathrm{R}}_{\mathcal{V}}(p)\right\|_{\mathrm{a}, \mathcal{V}} \lesssim\left(|\boldsymbol{\beta}|_{\sharp}^{\frac{1}{2}}+h^{\frac{1}{2}} \tau^{\frac{1}{2}}|\nabla \cdot \boldsymbol{\beta}|_{\sharp}\right) h^{\frac{1}{2}}|p|_{H^{1}(\Omega)},
$$

with stability constant $\varrho$ defined in Lemma 3.2, $|\boldsymbol{\beta}|_{\sharp}:=\|\boldsymbol{\beta}\|_{L^{\infty}(\Omega)}$, and $|\nabla \cdot \boldsymbol{\beta}|_{\sharp}:=\|\nabla \cdot \boldsymbol{\beta}\|_{L^{\infty}(\Omega)}$.

Proof. We need to bound the three terms in the right-hand side of (3.19) for all $\mathbf{q} \in \mathcal{V}$ such that $\|\mathbf{q}\|_{a, \mathcal{V}}=1$. A direct calculation shows that

$$
\llbracket \mathrm{q},\left\lfloor\mathrm{H}_{-\nabla \cdot \boldsymbol{\beta}}^{\mathcal{\nu}}, \widehat{\mathrm{R}}\right\rceil(p) \rrbracket_{\mathcal{\mathcal { C }}}=\sum_{v \in \mathrm{V}} \mathbf{q}_{v} \int_{\tilde{c}(v)}(-\nabla \cdot \boldsymbol{\beta})\left(p-\left(\widehat{\mathrm{R}}_{\mathcal{V}}(p)\right)_{v}\right) d \tilde{c}
$$

The definition of $\widehat{R}_{\mathcal{V}}$ together with the Poincaré inequality and the multiplicative trace inequality imply that

$$
\left\|q-\left(\widehat{\mathrm{R}}_{\mathcal{V}}(q)\right)_{v}\right\|_{L^{2}(\tilde{c}(v))}+h_{\tilde{c}(v)}^{\frac{1}{2}}\left\|q-\left(\widehat{\mathrm{R}}_{\mathcal{V}}(q)\right)_{v}\right\|_{L^{2}(\partial \tilde{c}(v))} \lesssim h_{\tilde{c}(v)}|q|_{H^{1}(\tilde{c}(v))},
$$

for all $q \in H^{1}(\Omega)$ and all $v \in \mathrm{V}$. Hence, we infer that

$$
\left|\llbracket \mathbf{q},\left\lfloor\mathbf{H}_{-\nabla \cdot \boldsymbol{\beta}}^{\mathcal{\nu}}, \widehat{\mathrm{R}}\right\rceil(p) \rrbracket_{\mathcal{\mathcal { C }}}\right| \lesssim\left(\tau^{-\frac{1}{2}}\|\mathbf{q}\|_{2, \mathcal{V}}\right)\left(h^{\frac{1}{2}} \tau^{\frac{1}{2}}|\nabla \cdot \boldsymbol{\beta}|_{\sharp}\right) h^{\frac{1}{2}}|p|_{H^{1}(\Omega)}
$$

Turning to the second term in (3.19), a direct calculation using $(\Lambda 1)$, the fact that $p$ is single-valued on 
$\tilde{f}(e)$, and recalling the definition of $\beta_{e}$ shows that

$$
-\llbracket \operatorname{GRAD}(\mathbf{q}),\left\lfloor L_{\boldsymbol{\beta}}^{\nu \widetilde{\mathcal{F}}}, \widehat{\mathrm{R}}\right\rceil(p) \rrbracket_{\mathcal{E} \tilde{\mathcal{F}}}=\sum_{e \in \mathrm{E}} \llbracket \mathrm{q} \rrbracket_{e} \sum_{v \in \mathrm{V}_{e}} \frac{1}{2}\left(1+\Lambda_{v e}^{\boldsymbol{\beta}}\right) \int_{\tilde{f}(e)} \boldsymbol{\beta} \cdot \boldsymbol{n}_{\tilde{f}(e)}\left(p-\left(\widehat{\mathrm{R}}_{\mathcal{V}}(p)\right)_{v}\right) d \tilde{f}
$$

whence we infer using (3.21) and the fact that $\left|\Lambda_{v e}\right| \leq 1$ that

$$
\left|\llbracket \operatorname{GRAD}(\mathbf{q}),\left\lfloor L_{\boldsymbol{\beta}}^{\mathcal{\mathcal { F }}}, \widehat{\mathrm{R}}\right\rceil(p) \rrbracket_{\mathcal{E} \tilde{\mathcal{F}}}\right| \lesssim\left(\sum_{e \in \mathrm{E}} \llbracket \mathrm{q} \rrbracket_{e}^{2} \int_{\tilde{f}(e)}\left|\boldsymbol{\beta} \cdot \boldsymbol{n}_{\tilde{f}(e)}\right| d \tilde{f}\right)^{\frac{1}{2}}|\boldsymbol{\beta}|_{\sharp}^{\frac{1}{2}} h^{\frac{1}{2}}|p|_{H^{1}(\Omega)} .
$$

Owing to the triangle inequality, Lemma 3.1 , and $(\Lambda 2)$, we infer that

$$
\left(\sum_{e \in \mathrm{E}} \llbracket \mathbf{q} \rrbracket_{e}^{2} \int_{\tilde{f}(e)}\left|\boldsymbol{\beta} \cdot \boldsymbol{n}_{\tilde{f}(e)}\right| d \tilde{f}\right)^{\frac{1}{2}} \leq c_{\Lambda}^{-\frac{1}{2}}\|\mathbf{q}\|_{\text {upw }, \boldsymbol{\beta}}+\left(\sum_{e \in \mathrm{E}} \llbracket \mathbf{q} \rrbracket_{e}^{2}\left(\int_{\tilde{f}(e)}\left|\boldsymbol{\beta} \cdot \boldsymbol{n}_{\tilde{f}(e)}\right| d \tilde{f}-\left|\beta_{e}\right|\right)\right)^{\frac{1}{2}} .
$$

Let $c \in \mathrm{C}_{e}$; the local dual face $\tilde{f}_{c}(e)$ consists of two triangles, say $\tilde{f}_{f, c}(e)$, each touching one of the two faces $f$ of $c$ sharing $e$. Set $\delta_{f, c}(e):=\int_{\tilde{f}_{f, c}(e)}\left|\boldsymbol{\beta} \cdot \boldsymbol{n}_{\tilde{f}_{f, c}(e)}\right| d \tilde{f}-\int_{\tilde{f}_{f, c}(e)} \boldsymbol{\beta} \cdot \boldsymbol{n}_{\tilde{f}_{f, c}(e)} d \tilde{f}$, so that

$$
0 \leq \delta_{f, c}(e)=2 \int_{\tilde{f}_{f, c}(e)}\left(\boldsymbol{\beta} \cdot \boldsymbol{n}_{\tilde{f}_{f, c}(e)}\right)^{-} d \tilde{f} .
$$

If $\left(\boldsymbol{\beta} \cdot \boldsymbol{n}_{\tilde{f}_{f, c}(e)}\right)^{-}$takes positive values on $\tilde{f}_{f, c}(e)$, then $0 \leq \delta_{f, c}(e) \leq 2\left|\beta_{e}\right|$; otherwise, $\boldsymbol{\beta} \cdot \boldsymbol{n}_{f_{f, c}(e)}$ vanishes at some point in $\tilde{f}_{f, c}(e)$. Then, using the fact that $\boldsymbol{\beta} \cdot \boldsymbol{n}_{\tilde{f}_{f, c}(e)}$ is Lipschitz in $\tilde{f}_{f, c}(e)$ together with mesh regularity leads to $0 \leq \delta_{f, c}(e) \lesssim L_{\beta} h_{e}\left|\tilde{f}_{f, c}(e)\right|$. Since $\left|\int_{\tilde{f}(e)}(\cdot)\right| \geq \int_{\tilde{f}(e)}(\cdot)$, summing these bounds over $c \in \mathrm{C}_{e}$ and the faces $f$ leads to

$$
0 \leq \int_{\tilde{f}(e)}\left|\boldsymbol{\beta} \cdot \boldsymbol{n}_{\tilde{f}(e)}\right| d \tilde{f}-\left|\int_{\tilde{f}(e)} \boldsymbol{\beta} \cdot \boldsymbol{n}_{\tilde{f}(e)} d \tilde{f}\right| \leq \sum_{f, c} \delta_{f, c}(e) \lesssim\left|\beta_{e}\right|+L_{\beta} h_{e}|\tilde{f}(e)| .
$$

Using the assumption $L_{\beta} \lesssim \tau^{-1}$, mesh regularity, and the definition of the discrete norm $\|\cdot\|_{2, \mathcal{V}}$ leads to

$$
\left(\sum_{e \in \mathrm{E}} \llbracket \mathrm{q} \rrbracket_{e}^{2} \int_{\tilde{f}(e)}\left|\boldsymbol{\beta} \cdot \boldsymbol{n}_{\tilde{f}(e)}\right| d \tilde{f}\right)^{\frac{1}{2}} \lesssim\|\mathrm{q}\|_{\mathrm{upw}, \boldsymbol{\beta}}+\tau^{-\frac{1}{2}}\|\mathbf{q}\|_{2, \mathcal{V}} .
$$

Finally, a direct calculation shows that

$$
\llbracket \mathrm{q}^{\partial},\left\lfloor\mathrm{H}_{(\boldsymbol{\beta} \cdot \boldsymbol{n})^{+}}^{\partial}, \widehat{\mathrm{R}}\right\rceil(p) \rrbracket_{(\nu \tilde{\mathcal{F}})^{\partial}}=\sum_{v \in \mathrm{V}^{\partial}} \mathbf{q}_{v} \int_{\tilde{f}^{\partial}(v)}(\boldsymbol{\beta} \cdot \boldsymbol{n})^{+}\left(p-\left(\widehat{\mathrm{R}}_{\mathcal{V}}(p)\right)_{v}\right) d \tilde{f}^{\partial}
$$

so that $\left|\llbracket \mathbf{q}^{\partial},\left\lfloor\mathbf{H}_{(\boldsymbol{\beta} \cdot \boldsymbol{n})^{+}}^{\partial}, \widehat{\mathrm{R}}\right\rceil(p) \rrbracket_{(\nu \tilde{\mathcal{F}})^{\partial}}\right| \lesssim\|\mathbf{q}\|_{|\boldsymbol{\beta} \cdot \boldsymbol{n}|}|\boldsymbol{\beta}|_{\sharp}^{\frac{1}{2}} h^{\frac{1}{2}}|p|_{H^{1}(\Omega)}$. This completes the proof.

Remark 3.6 (Localization). The error estimate (3.20) can be localized to dual mesh cells.

\section{Advection-diffusion}

This section addresses the derivation and analysis of vertex-based CDO schemes for the advection-diffusion problem (1.1). The diffusion tensor $\boldsymbol{\lambda}$ takes symmetric, uniformly positive definite values. For simplicity, 
we assume that $\boldsymbol{\lambda}$ is constant in each primal cell $c \in \mathrm{C}$ with minimal and maximal eigenvalues $\lambda_{b, c}$ and $\lambda_{\sharp, c}$, respectively, and local anisotropy ratio $\rho_{c}=\lambda_{\sharp, c} / \lambda_{b, c} \geq 1$. The analysis can be extended to locally Lipschitz diffusion tensors.

\subsection{Preliminaries: boundary penalty for pure diffusion}

In this section, we consider the pure-diffusion version of the model problem (1.1) with $\boldsymbol{\beta} \equiv \mathbf{0}$ :

$$
\begin{aligned}
& -\nabla \cdot(\boldsymbol{\lambda} \nabla p)=s \quad \text { a.e. in } \Omega, \\
& p=p_{\mathrm{D}} \quad \text { a.e. on } \partial \Omega .
\end{aligned}
$$

Formally, the weak formulation is as follows: For all $q \in H^{1}(\Omega)$,

$$
\int_{\Omega} \nabla q \cdot \boldsymbol{\lambda} \cdot \nabla p d \Omega-\int_{\partial \Omega} q(\boldsymbol{n} \cdot \boldsymbol{\lambda} \cdot \nabla p) d \partial \Omega+\int_{\partial \Omega} \eta q p d \partial \Omega=\int_{\Omega} q s d \Omega+\int_{\partial \Omega} \eta q p_{\mathrm{D}} d \partial \Omega,
$$

with some boundary penalty parameter $\eta$. It is also possible to consider a symmetric bilinear form on the left-hand side. Symmetry is an important property when invoking duality arguments for pure diffusion problems; it is also a relevant property when inverting the linear system. It is less important in the presence of advection.

\subsubsection{CDO scheme}

The vertex-based CDO scheme with weakly enforced boundary conditions is formulated in terms of a discrete Hodge operator $\mathrm{H}_{\boldsymbol{\lambda}}^{\tilde{\mathcal{F}}}: \mathcal{E} \rightarrow \widetilde{\mathcal{F}}$, which is the discrete counterpart of the map $\boldsymbol{g} \mapsto \boldsymbol{\lambda} \cdot \boldsymbol{g}$, and the discrete boundary operators $\mathrm{N}_{\lambda}^{\partial}: \mathcal{E} \rightarrow \widetilde{\mathcal{F}}^{\partial}$ (normal flux) and $\widehat{\mathrm{H}}_{\lambda / h}^{\partial}: \mathcal{V}^{\partial} \rightarrow \widetilde{\mathcal{F}}^{\partial}$ (boundary penalty), which weakly enforce boundary conditions à la Nitsche and which are the discrete counterparts of the maps $\boldsymbol{g} \mapsto \boldsymbol{n} \cdot \boldsymbol{\lambda} \cdot \boldsymbol{g}$ and $p \mapsto(\lambda / h) p$ at the boundary, respectively. The discrete problem consists in finding $\mathrm{p} \in \mathcal{V}$ such that

$$
\mathrm{A}_{\boldsymbol{\lambda}}(\mathrm{p}, \mathrm{q})=\llbracket \mathrm{q}, \mathrm{s} \rrbracket_{\mathcal{\mathcal { V }} \tilde{\mathcal{C}}}+\llbracket \mathrm{q}^{\partial}, \phi_{\mathrm{D}} \rrbracket_{(\mathcal{V} \tilde{\mathcal{F}})}{ }^{\partial}, \quad \forall \mathrm{q} \in \mathcal{V}
$$

with bilinear form such that

$$
\mathrm{A}_{\boldsymbol{\lambda}}(\mathrm{p}, \mathrm{q}):=\llbracket \operatorname{GRAD}(\mathrm{q}), \mathrm{H}_{\lambda}^{\mathcal{E} \tilde{\mathcal{F}}} \operatorname{GRAD}(\mathrm{p}) \rrbracket_{\mathcal{E} \tilde{\mathcal{F}}}-\llbracket \mathrm{q}^{\partial}, \mathrm{N}_{\lambda}^{\partial} \operatorname{GRAD}(\mathrm{p}) \rrbracket_{(\nu \tilde{\mathcal{F}})^{\partial}}+\eta_{0} \llbracket \mathrm{q}^{\partial}, \widehat{\mathrm{H}}_{\lambda / h}^{\partial}\left(\mathrm{p}^{\partial}\right) \rrbracket_{(\nu \tilde{\mathcal{F}})^{\partial}},
$$

where $\eta_{0}>0$ is a real number to be chosen large enough (see below), $\mathbf{s}=\mathrm{R}_{\widetilde{\mathcal{C}}}(s)$, and $\phi_{\mathrm{D}}=\eta_{0} \widehat{\mathrm{H}}_{\lambda / h}^{\partial}\left(\mathrm{R}_{\mathcal{V}^{\partial}}\left(p_{\mathrm{D}}\right)\right)$. The bilinear form (4.3) extends that of [9] where the Dirichlet boundary condition was strongly enforced.

The discrete Hodge operator $\mathrm{H}_{\lambda}^{\tilde{\mathcal{F}}}$ is assembled cell-wise from local operators $\mathrm{H}_{\lambda}^{\left(\mathcal{E} \tilde{\mathcal{F}}_{c}\right.}: \mathcal{E}_{c} \rightarrow \widetilde{\mathcal{F}}_{c}$ for all $c \in \mathrm{C}$, so that

$$
\llbracket \mathrm{g}_{1}, \mathrm{H}_{\lambda}^{\tilde{\mathcal{F}}}\left(\mathrm{g}_{2}\right) \rrbracket_{\mathcal{E} \tilde{\mathcal{F}}}=\sum_{c \in \mathrm{C}} \llbracket \mathrm{g}_{1}, \mathrm{H}_{\lambda}^{(\mathcal{( \mathcal { \mathcal { F } }})_{c}}\left(\mathrm{~g}_{2}\right) \rrbracket_{(\mathcal{E} \tilde{\mathcal{F}})_{c}}
$$

for all $\mathrm{g}_{1}, \mathrm{~g}_{2} \in \mathcal{E}$. Similarly, the discrete normal flux operator $\mathrm{N}_{\lambda}^{\partial}$ is assembled face-wise from local operators $\mathrm{N}_{\lambda}^{\partial_{f}}: \mathcal{E}_{c} \rightarrow \widetilde{\mathcal{F}}_{f}^{\partial}$ for all $f \in \mathrm{F}^{\partial}$, where $c=c(f)$ is the primal cell containing the primal boundary face $f$, so that

$$
\llbracket \mathrm{q}^{\partial}, \mathrm{N}_{\lambda}^{\partial}(\mathrm{g}) \rrbracket_{(\mathcal{\nu} \tilde{\mathcal{F}})^{\partial}}=\sum_{f \in \mathrm{F}^{\partial}} \llbracket \mathrm{q}^{\partial}, \mathrm{N}_{\lambda}^{\partial f}(\mathrm{~g}) \rrbracket_{(\nu \tilde{\mathcal{F}})_{f}^{\partial}},
$$

for all $\mathrm{q}^{\partial} \in \mathcal{V}^{\partial}$ and all $\mathrm{g} \in \mathcal{E}$. Note that this implies that $\mathrm{N}_{\lambda}^{\partial}(\mathrm{g})$, for all $\mathrm{g} \in \mathcal{E}$, only depends on the 
components of $\mathrm{g}$ attached to an edge of a cell having a boundary face. The discrete boundary penalty operator $\widehat{\mathrm{H}}_{\lambda / h}^{\partial}$ is such that, still with $c=c(f)$,

$$
\widehat{\mathrm{H}}_{\lambda / h}^{\partial}\left(\mathrm{q}^{\partial}\right)_{\tilde{f}^{\partial}(v)}:=\mathrm{q}_{v}^{\partial} \sum_{f \in \mathrm{F}_{v}^{\partial}}\left|\tilde{f}_{f}^{\partial}(v)\right| \lambda_{\sharp, c} h_{c}^{-1},
$$

for all $\mathrm{q}^{\partial} \in \mathcal{V}^{\partial}$ and all $v \in \mathrm{V}^{\partial}$, where $\mathrm{F}_{v}^{\partial}:=\left\{f \in \mathrm{F}^{\partial} \mid v \in f\right\}$. Note that $\widehat{\mathrm{H}}_{\lambda / h}^{\partial}$ is algebraically represented by a diagonal matrix.

\subsubsection{Example}

Let us give a concrete example of CDO scheme. We consider a reconstruction operator $\mathbf{L}_{\mathcal{E}_{c}}: \mathcal{E}_{c} \rightarrow \boldsymbol{L}^{\infty}(c)$ for all $c \in \mathrm{C}$. The discrete Hodge operator in each cell $c \in \mathrm{C}$ is defined such that

$$
\llbracket \mathrm{g}_{1}, \mathrm{H}_{\lambda}^{(\mathcal{E} \tilde{\mathcal{F}})}\left(\mathrm{g}_{2}\right) \rrbracket_{(\mathcal{E} \tilde{\mathcal{F}})}=\int_{c} \mathbf{L}_{\mathcal{E}_{c}}\left(\mathrm{~g}_{1}\right) \cdot \boldsymbol{\lambda} \cdot \mathbf{L}_{\mathcal{E}_{c}}\left(\mathrm{~g}_{2}\right) d c,
$$

for all $\mathrm{g}_{1}, \mathrm{~g}_{2} \in \mathcal{E}_{c}$, while the discrete normal flux operator in each boundary face $f \in \mathrm{F}^{\partial}$ is defined as follows (with $c=c(f)$ the primal cell containing $f$ ):

$$
\mathrm{N}_{\lambda}^{\partial_{f}}(\mathrm{~g})_{\tilde{f}_{f}^{\partial}(v)}:=\int_{\tilde{f}_{f}^{\partial}(v)} \boldsymbol{n} \cdot \boldsymbol{\lambda} \cdot \mathbf{L}_{\mathcal{E}_{c}}(\mathrm{~g}) d \tilde{f}
$$

for all $v \in \mathrm{V}_{f}^{\partial}$ and all $\mathrm{g} \in \mathcal{E}_{c}$. The reconstruction operator has to satisfy some properties stated in Lemma 4.1 below. One possibility is to consider the reconstruction proposed by Codecasa et al. [16], see also [9, 8], whereby $\mathbf{L}_{\mathcal{E}_{c}}(\mathrm{~g})$ is piecewise constant on each diamond $\mathfrak{d}(e) \cap c, e \in \mathrm{E}_{c}$ (see Figure 2, left panel).

\subsubsection{Design conditions}

More generally, the design conditions on $\mathrm{H}_{\lambda}^{(\mathcal{E} \tilde{\mathcal{F}}) c}$ are as follows: For all $c \in \mathrm{C}$,

(H1) [Stability] $\mathrm{H}_{\lambda}^{(\tilde{\mathcal{F}})_{c}}$ is self-adjoint and monotone, and there exists $c_{\mathrm{H}}>0$, uniform with respect to the mesh and the model parameters, such that, for all $\mathrm{g} \in \mathcal{E}_{c}$,

$$
c_{\mathrm{H}} \lambda_{b, c}\|\mathrm{~g}\|_{2, \mathcal{E}_{c}}^{2} \leq \llbracket \mathrm{g}, \mathrm{H}_{\lambda}^{(\tilde{\mathcal{F}})_{c}}(\mathrm{~g}) \rrbracket_{(\mathcal{E} \tilde{\mathcal{F}})_{c}} \leq c_{\mathrm{H}}^{-1} \lambda_{\sharp, c}\|\mathrm{~g}\|_{2, \mathcal{E}_{c}}^{2} .
$$

(H2) $\left[\mathbb{P}_{0}\right.$-consistency] $\mathrm{R}_{\widetilde{\mathcal{F}}_{c}}(\boldsymbol{\lambda} \cdot \boldsymbol{G})=\mathrm{H}_{\boldsymbol{\lambda}}^{(\tilde{\mathcal{F}}) c_{c}}\left(\mathrm{R}_{\mathcal{E}_{c}}(\boldsymbol{G})\right)$ for any constant field $\boldsymbol{G}$ in $c$.

The design conditions on $\mathrm{N}_{\lambda}^{\partial_{f}}$ are as follows: For all $f \in \mathrm{F}^{\partial}$, with $c=c(f)$,

(N1) [Boundedness] There exists $c_{\mathrm{N}}$, uniform with respect to the mesh and the model parameters, such that, for all $\mathrm{g} \in \mathcal{E}_{c}$,

$$
\sum_{v \in \mathrm{V}_{f}^{\partial}}\left|\tilde{f}_{f}^{\partial}(v)\right|^{-1}\left(\left(\mathrm{~N}_{\lambda}^{\partial_{f}}(\mathrm{~g})\right)_{\tilde{f}_{f}^{\partial}(v)}\right)^{2} \leq c_{\mathrm{N}} \lambda_{\sharp, c} h_{\sharp, c}^{-1} \llbracket \mathrm{g}, \mathrm{H}_{\lambda}^{(\mathcal{E} \tilde{\mathcal{F}})_{c}}(\mathrm{~g}) \rrbracket_{(\mathcal{\mathcal { F }})_{c}} .
$$

(N2) $\left[\mathbb{P}_{0}\right.$-consistency $] \mathrm{R}_{\widetilde{\mathcal{F}}_{f}^{\partial}}(\boldsymbol{n} \cdot \boldsymbol{\lambda} \cdot \boldsymbol{G})=\mathrm{N}_{\boldsymbol{\lambda}}^{\partial_{f}}\left(\mathrm{R}_{\mathcal{E}_{c}}(\boldsymbol{G})\right)$ for any constant field $\boldsymbol{G}$ in $c$.

Lemma 4.1 (Design conditions). Let the discrete Hodge and normal flux operators be defined by (4.8) and (4.9), respectively. Assume that the reconstruction operator is such that: 
(i) [Stability] $\mathbf{L}_{\mathcal{E}_{c}}$ (g) is a piecewise-polynomial function in c, and there exists $c_{\mathrm{L}}>0$, uniform with respect to the mesh-size, such that $c_{\mathrm{L}}\|\mathrm{g}\|_{2, \mathcal{E}_{c}}^{2} \leq\left\|\mathbf{L}_{\mathcal{E}_{c}}(\mathrm{~g})\right\|_{L^{2}(c)}^{2} \leq c_{\mathrm{L}}^{-1}\|\mathrm{~g}\|_{2, \mathcal{E}_{c}}^{2}$ for all $\mathrm{g} \in \mathcal{E}_{c}$.

(ii) [Partition of unity] $\mathbf{L}_{\mathcal{E}_{c}}\left(\mathrm{R}_{\mathcal{E}_{c}}(\boldsymbol{G})\right)=\boldsymbol{G}$ for any constant field $\boldsymbol{G}$ in c.

(iii) [Dual consistency] $\int_{c} \mathbf{L}_{\mathcal{E}_{c}}(\mathrm{~g}) d c=\sum_{e \in \mathcal{E}_{c}}\left(\int_{\tilde{f}_{c}(e)} \boldsymbol{n}_{\tilde{f}_{c}(e)} d \tilde{f}\right) \mathrm{g}_{e}$ for all $\mathrm{g} \in \mathcal{E}_{c}$.

Then, (H1)-(H2) and (N1)-(N2) hold.

Proof. For the proof of (H1)-(H2), see $[9,8,16]$. To prove (N1), fix $f \in \mathrm{F}^{\partial}$ and observe that

$$
\begin{aligned}
\sum_{v \in \mathrm{V}_{f}^{\partial}}\left|\tilde{f}_{f}^{\partial}(v)\right|^{-1}\left(\left(\mathrm{~N}_{\boldsymbol{\lambda}}^{\partial_{f}}(\mathrm{~g})\right)_{\tilde{f}_{f}^{\partial}(v)}\right)^{2} & =\sum_{v \in \mathrm{V}_{f}^{\partial}}\left|\tilde{f}_{f}^{\partial}(v)\right|^{-1}\left(\int_{\tilde{f}_{f}^{\partial}(v)} \boldsymbol{n} \cdot \boldsymbol{\lambda} \cdot \mathbf{L}_{\mathcal{E}_{c}}(\mathrm{~g}) d \tilde{f}\right)^{2} \\
& \leq \sum_{v \in \mathrm{V}_{f}^{\partial}} \lambda_{\sharp, c}\left\|\boldsymbol{\lambda}^{1 / 2} \mathbf{L}_{\mathcal{E}_{c}}(\mathrm{~g})\right\|_{L^{2}\left(\tilde{f}_{f}^{\partial}(v)\right)}^{2} \\
& \leq c_{\mathrm{tr}} \sum_{v \in \mathrm{V}_{f}^{\partial}} \lambda_{\sharp, c} h_{\sharp, c}^{-1}\left\|\boldsymbol{\lambda}^{1 / 2} \mathbf{L}_{\mathcal{E}_{c}}(\mathrm{~g})\right\|_{L^{2}(c)}^{2} \\
& =c_{\mathrm{tr}} \#\left(\mathrm{~V}_{f}^{\partial}\right) \lambda_{\sharp, c} h_{\sharp, c}^{-1} \llbracket \mathrm{g}, \mathrm{H}_{\lambda}^{(\mathcal{\varepsilon} \tilde{\mathcal{F}})_{c}}(\mathrm{~g}) \rrbracket_{(\mathcal{\varepsilon} \tilde{\mathcal{F}})_{c}},
\end{aligned}
$$

where we have used the Cauchy-Schwarz inequality followed by a discrete trace inequality with $c=c(f)$ (since $\boldsymbol{\lambda}$ is constant and $\mathbf{L}_{\mathcal{E}_{c}}(\mathrm{~g})$ is a piecewise-polynomial) and the definition of the discrete Hodge operator. This proves $(\mathbf{N} 1)$ with $c_{\mathrm{N}}=c_{\mathrm{tr}} \#\left(\mathrm{~V}_{f}^{\partial}\right)$ (observing that the cardinal number $\#\left(\mathrm{~V}_{f}^{\partial}\right)$ is uniformly bounded owing to mesh regularity). Finally, letting $\boldsymbol{G}$ be a constant field in $c$, (N2) follows from

$$
\mathrm{N}_{\boldsymbol{\lambda}}^{\partial f}\left(\mathrm{R}_{\mathcal{E}_{c}}(\boldsymbol{G})\right)_{\tilde{f}_{f}^{\partial}(v)}=\int_{\tilde{f}_{f}^{\partial}(v)} \boldsymbol{n} \cdot \boldsymbol{\lambda} \cdot \mathbf{L}_{\mathcal{E}}\left(\mathrm{R}_{\mathcal{E}_{c}}(\boldsymbol{G})\right) d \tilde{f}=\int_{\tilde{f}_{f}^{\partial}(v)} \boldsymbol{n} \cdot \boldsymbol{\lambda} \cdot \boldsymbol{G} d \tilde{f}=\left(\mathrm{R}_{\widetilde{\mathcal{F}}_{f}^{\partial}}(\boldsymbol{n} \cdot \boldsymbol{\lambda} \cdot \boldsymbol{G})\right)_{\tilde{f}_{f}^{\partial}(v)},
$$

for all $v \in \mathrm{V}_{f}^{\partial}$ owing to property (ii) of the reconstruction operator.

\subsubsection{Analysis}

This section collects the main results concerning the analysis of the CDO scheme with boundary penalty. To facilitate the reading, the proofs are postponed to Section 7 . We define the following norms on $\mathcal{E}$ and $\mathcal{V}^{\partial}$, respectively:

$$
\|\mathrm{g}\|_{\lambda}^{2}:=\llbracket \mathrm{g}, \mathrm{H}_{\lambda}^{\mathcal{E} \tilde{\mathcal{F}}}(\mathrm{g}) \rrbracket_{\mathcal{E} \tilde{\mathcal{F}}}, \quad\left\|\mathrm{q}^{\partial}\right\|_{\lambda / h}^{2}=\llbracket \mathrm{q}^{\partial}, \widehat{\mathrm{H}}_{\lambda / h}^{\partial}\left(\mathrm{q}^{\partial}\right) \rrbracket_{(\nu \tilde{\mathcal{F}}) \partial} \cdot
$$

Observe that these norms can be localized as $\|\mathrm{g}\|_{\boldsymbol{\lambda}}^{2}=\sum_{c \in \mathrm{C}}\left\|\mathrm{g}_{c}\right\|_{\boldsymbol{\lambda}, c}^{2}$ with $\left\|\mathrm{g}_{c}\right\|_{\boldsymbol{\lambda}, c}^{2}=\llbracket \mathrm{g}_{c}, \mathrm{H}_{\lambda}^{(\mathcal{\varepsilon} \tilde{\mathcal{F}})_{c}}\left(\mathrm{~g}_{c}\right) \rrbracket_{(\mathcal{\varepsilon} \tilde{\mathcal{F}})_{c}}$ for all $\mathrm{g}_{c} \in \mathcal{E}_{c}$, and as $\left\|\mathbf{q}^{\partial}\right\|_{\lambda / h}^{2}=\sum_{f \in \mathrm{F}^{\partial}}\left\|\mathbf{q}_{f}^{\partial}\right\|_{\lambda / h, f}^{2}$ with $\left\|\mathbf{q}_{f}^{\partial}\right\|_{\lambda / h, f}^{2}=\lambda_{\sharp, c} h_{c}^{-1} \sum_{v \in \mathrm{V}_{f}^{\partial}}\left|\tilde{f}_{f}^{\partial}(v)\right|\left(\mathbf{q}_{f, v}^{\partial}\right)^{2}$ for all $\mathbf{q}_{f}^{\partial} \in \mathcal{V}_{f}^{\partial}$. The stability of the CDO scheme (4.3) is expressed in the following norm:

$$
\|\mathbf{q}\|_{\mathrm{d}, \mathcal{V}}^{2}:=\|\operatorname{GRAD}(\mathbf{q})\|_{\lambda}^{2}+\left\|\mathbf{q}^{\partial}\right\|_{\lambda / h}^{2}, \quad \forall \mathbf{q} \in \mathcal{V}
$$

Lemma 4.2 (Coercivity and well-posedness). Assume (H1) and (N1). Then, provided $\eta_{0} \geq 1+\frac{1}{2} c_{\mathrm{N}}^{2}$, the following holds:

$$
\varrho\|q\|_{d, \mathcal{V}}^{2} \leq A_{\lambda}(\mathbf{q}, q), \quad \forall \mathbf{q} \in \mathcal{V},
$$

with $\varrho=\frac{1}{2}$. Consequently, (4.3) is well-posed. 
We now address the consistency of the scheme (4.3). We assume that the exact solution $p$ is in $H^{s}(\Omega)$, $s>\frac{3}{2}$ (the regularity assumption can be localized to mesh cells), and we consider the (classical) de Rham map $R_{\mathcal{V}}$. We define the following two commutators:

$$
\begin{aligned}
\left\lfloor\mathrm{H}_{\boldsymbol{\lambda}}^{\mathcal{E}}, \mathrm{R}\right\rceil(\nabla p) & :=\mathrm{R}_{\widetilde{\mathcal{F}}}(\boldsymbol{\lambda} \cdot \nabla p)-\mathrm{H}_{\boldsymbol{\lambda}}^{\mathcal{E \mathcal { F }}}\left(\operatorname{GRAD}\left(\mathrm{R}_{\mathcal{V}}(p)\right)\right), \\
\left\lfloor\mathrm{N}_{\boldsymbol{\lambda}}^{\partial}, \mathrm{R}\right\rceil(\nabla p) & :=\mathrm{R}_{\widetilde{\mathcal{F}} \partial}(\boldsymbol{n} \cdot \boldsymbol{\lambda} \cdot \nabla p)-\mathrm{N}_{\boldsymbol{\lambda}}^{\partial}\left(\operatorname{GRAD}\left(\mathrm{R}_{\mathcal{V}}(p)\right)\right) .
\end{aligned}
$$

Lemma 4.3 (Consistency). Let $p$ be the unique solution of (4.1) and let $\mathrm{p}$ be the unique solution of (4.3). Assume $p \in H^{s}(\Omega), s>\frac{3}{2}$. Then, under the assumptions of Lemma 4.2, the following holds:

$$
\varrho\left\|\mathrm{p}-\mathrm{R}_{\mathcal{V}}(p)\right\|_{\mathrm{d}, \mathcal{V}} \leq \sup _{\mathbf{q} \in \mathcal{V} ;\|\boldsymbol{q}\|_{\mathrm{d}, \mathcal{V}=1}} \mathrm{E}_{\boldsymbol{\lambda}}(p, \mathbf{q})
$$

with consistency error

$$
\mathrm{E}_{\lambda}(p, \mathrm{q}):=\llbracket \operatorname{GRAD}(\mathrm{q}),\left\lfloor\mathrm{H}_{\lambda}^{\mathcal{E} \tilde{\mathcal{F}}}, \mathrm{R}\right\rceil(\nabla p) \rrbracket_{\mathcal{E} \tilde{\mathcal{F}}}-\llbracket \mathrm{q}^{\partial},\left\lfloor\mathrm{N}_{\lambda}^{\partial}, \mathrm{R}\right\rceil(\nabla p) \rrbracket_{(\nu \tilde{\mathcal{F}})^{\partial}} \cdot
$$

Theorem 4.4 (Convergence rate). Let $p$ be the unique solution of (4.1) and let $\mathrm{p}$ be the unique solution of (4.3). Assume (H1)-(H2) and (N1)-(N2). Assume $p \in H^{2}(\Omega)$. Then, the following holds:

$$
\left\|\mathrm{p}-\mathrm{R}_{\mathcal{V}}(p)\right\| \|_{\mathrm{d}, \mathcal{V}} \lesssim\left(\sum_{c \in \mathrm{C}} \rho_{c} \lambda_{\sharp, c} h_{c}^{2}|p|_{H^{2}(c)}^{2}\right)^{\frac{1}{2}} .
$$

\subsection{CDO Scheme for advection-diffusion}

Vertex-based CDO schemes for the advection-diffusion problem (1.1) hinge on the discrete bilinear form $\mathrm{A}_{\beta, \lambda}:=\mathrm{A}_{\beta}+\mathrm{A}_{\boldsymbol{\lambda}}$ with $\mathrm{A}_{\boldsymbol{\beta}}$ defined by (3.5) and $\mathrm{A}_{\boldsymbol{\lambda}}$ by (4.4). The discrete problem consists in finding $\mathrm{p} \in \mathcal{V}$ such that

$$
\mathrm{A}_{\beta, \lambda}(\mathrm{p}, \mathrm{q})=\llbracket \mathrm{q}, \mathrm{s} \rrbracket_{\mathcal{V} \tilde{\mathcal{C}}}+\llbracket \mathrm{q}^{\partial}, \phi_{\mathrm{D}} \rrbracket_{(\mathcal{V} \tilde{\mathcal{F}})^{\partial}}, \quad \forall \mathrm{q} \in \mathcal{V},
$$

with $\mathrm{s}=\mathrm{R}_{\widetilde{\mathcal{C}}}(s)$ and $\phi_{\mathrm{D}}=\mathrm{R}_{\widetilde{\mathcal{F}}^{\partial}}\left((\boldsymbol{\beta} \cdot \boldsymbol{n})^{-} p_{\mathrm{D}}\right)+\eta_{0} \widehat{\mathrm{H}}_{\lambda / h}^{\partial}\left(\mathrm{R}_{\mathcal{V}^{\partial}}\left(p_{\mathrm{D}}\right)\right)$. The Tonti diagram of the vertex-based CDO scheme (4.19) is presented in Figure 4. Variants, such as using the conservative form of the advective derivative or including a reactive term, can be considered as well; see Remarks 3.2 and 3.3.

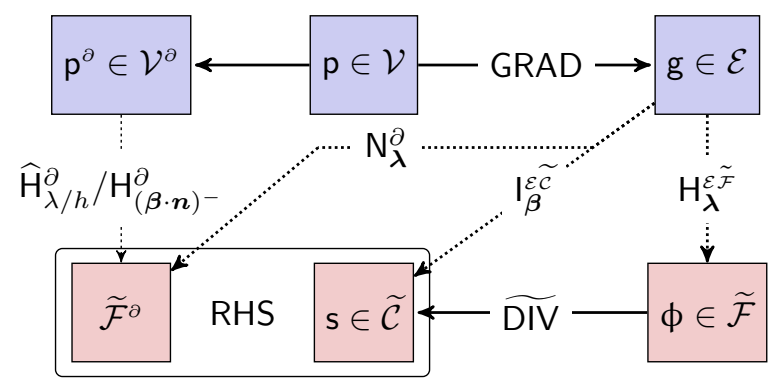

Figure 4: Tonti diagram of the vertex-based CDO scheme for advection-diffusion with weakly enforced boundary conditions

We define the stability norm on $\mathcal{V}$ as $\|\mathrm{q}\|_{\text {ad, } \mathcal{V}}^{2}:=\|\mathrm{q}\|_{\mathrm{a}, \mathcal{V}}^{2}+\|\mathrm{q}\|_{\mathrm{d}, \mathcal{V}}^{2}$ with advection-related stability norm defined by (3.14) and diffusion-related stability norm defined by (4.13). 


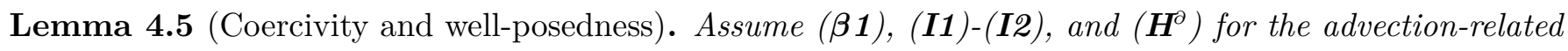
terms together with $(\boldsymbol{H} \mathbf{1})$ and $(\boldsymbol{N 1})$ for the diffusion-related terms. Then, provided $\eta_{0} \geq 1+\frac{1}{2} c_{\mathrm{N}}^{2}$, the following holds:

$$
\varrho\|\mathrm{q}\|_{\mathrm{ad}, \mathcal{V}}^{2} \leq \mathrm{A}_{\beta, \lambda}(\mathrm{q}, \mathrm{q}), \quad \forall \mathrm{q} \in \mathcal{V}
$$

with $\varrho=\frac{1}{2}$. Consequently, (4.19) is well-posed.

Proof. Combine Lemma 3.2 with Lemma 4.2 .

Lemma 4.6 (Error bound). Let $p$ be the unique solution of (1.1) and let $\mathrm{p}$ be the unique solution of (4.19). Assume $p \in H^{s}(\Omega), s>\frac{3}{2}$. Then, under the assumptions of Lemma 4.5, the following holds:

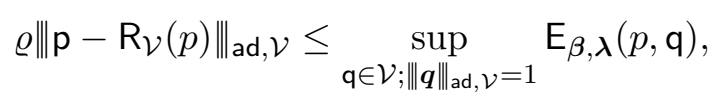

with consistency error $\mathrm{E}_{\boldsymbol{\beta}, \boldsymbol{\lambda}}(p, \mathrm{q})=\mathrm{E}_{\boldsymbol{\beta}}(p, \mathrm{q})+\mathrm{E}_{\boldsymbol{\lambda}}(p, \mathrm{q})$, with $\mathrm{E}_{\boldsymbol{\beta}}(p, \mathrm{q})$ defined by (3.19) with $\mathrm{R}_{\mathcal{V}}$ in lieu of $\widehat{\mathrm{R}}_{\mathcal{V}}$, and $\mathrm{E}_{\boldsymbol{\lambda}}(p, \mathrm{q})$ defined by (4.17).

Proof. Combine Lemma 3.3 with Lemma 4.3 (note that $H^{1}(\Omega) \subset V_{\beta}(\Omega)$ ).

\subsection{Example: CDO scheme with Péclet-based upwinding}

For all $e \in \mathrm{E}$, we define the (algebraic) edge Péclet number as $\mathrm{Pe}_{e}=\lambda_{e}^{-1}|f(e)|^{-1} \beta_{e} h_{e}$ with $\lambda_{e}=\max _{c \in \mathrm{C}_{e}} \lambda_{b, c}$, $\mathrm{C}_{e}=\{c \in \mathrm{C} \mid e \subset \partial c\}$, and $\beta_{e}$ defined in Section 3.2. We then use (3.10)-(3.11) to define the discrete contraction operators $\left.\right|_{\beta} ^{\mathcal{E} \widetilde{\mathcal{C}}}$ and $\mathbf{I}_{\beta}^{\mathcal{F} \widetilde{\mathcal{F}}}$ with Péclet-dependent upwinding parameter $\Lambda_{v e}=\Theta\left(\iota_{\tilde{f}(e), \tilde{c}(v)} P \mathrm{Pe}_{e}\right)$, where the function $\Theta: \mathbb{R} \rightarrow \mathbb{R}$ is such that

$(\Theta 1) \Theta(x)+\Theta(-x)=0$ and $\Theta(x) \geq 0$ for all $x \in \mathbb{R}_{\geq 0}$.

( $\Theta 2)$ There exists $\alpha>0$ such that $\Theta(x) \geq \alpha$ for all $x \geq 1$ (the lower bound on $x$ is arbitrary; changing its value only changes the constants in the error bounds).

Note that $(\Theta 1)$ implies $(\Lambda 1)$ since $\beta_{e} \Lambda_{e}=\frac{1}{2} \frac{\lambda_{e}|\tilde{f}(e)|}{h_{e}} \sum_{v \in \mathrm{V}_{e}} \iota_{\tilde{f}(e), \tilde{c}(v)} \mathrm{Pe}_{e} \Theta\left(\iota_{\tilde{f}(e), \tilde{c}(v)} \mathrm{Pe}_{e}\right) \geq 0$. Since $(\Lambda 1)$ holds, Lemma 3.1 implies that (I1)-(I2) hold; hence, stability and well-posedness hold owing to Lemma 4.5. An example for the function $\Theta$ is the Sharfetter-Gummel map $\Theta(x)=\operatorname{coth}\left(\frac{x}{2}\right)-\frac{2}{x}$, see Roos et al. [35] for further insight and examples. The function $\Theta$ is related to the function $|A|$ introduced in [19] in the context of high-order face-based discretizations by the relation $|A|(x)=x \Theta(x)$.

To write the error estimate, we introduce one last geometric object $\mathfrak{d}(e)$, for all $e \in \mathrm{E}$, which is the so-called diamond around $e$ formed by the two pyramids of apex $v \in \mathrm{V}_{e}$ and (non-planar) basis $\tilde{f}(e)$, see Figure 2 (left panel). Note that $\cup_{e \in \mathrm{E}} \mathfrak{d}(e)=\Omega$.

Theorem 4.7 (Convergence rate). Assume $(\boldsymbol{\beta} 1)$. Let $L_{\beta}$ be the Lipschitz constant of $\boldsymbol{\beta}$ and assume that $L_{\beta} \lesssim \tau^{-1}$. Let the discrete contraction and surface Hodge operators be given by (3.10)-(3.11)-(3.12). For the diffusion-related operators, assume (H1)-(H2) and (N1)-(N2). Let $p$ be the unique solution of (1.1) 
and let $\mathrm{p}$ be the unique solution of (4.19). Assume $p \in H^{2}(\Omega)$. Then, the following holds:

$$
\begin{aligned}
\varrho\left\|\mathrm{p}-\mathrm{R}_{\mathcal{V}}(p)\right\|_{\mathrm{ad}, \mathcal{V}} \lesssim & \left(\sum_{c \in \mathrm{C}} \rho_{c} \lambda_{\sharp, c} h_{c}^{2}|p|_{H^{2}(c)}^{2}\right)^{\frac{1}{2}} \\
& +\left(\sum_{e \in \mathrm{E}}\left(\tau|\nabla \cdot \boldsymbol{\beta}|_{L^{\infty}(\mathfrak{d}(e))}^{2} h_{e}+|\boldsymbol{\beta} \cdot \boldsymbol{n}|_{\sharp, e}\right) h_{e} \min \left(1, \mathrm{Pe}_{e}\right)|p|_{H^{1+(\mathfrak{d}(e))}}^{2}\right)^{\frac{1}{2}},
\end{aligned}
$$

with $|\boldsymbol{\beta} \cdot \boldsymbol{n}|_{\sharp, e}:=\left\|\boldsymbol{\beta} \cdot \boldsymbol{n}_{\tilde{f}(e)}\right\|_{L^{\infty}(\tilde{f}(e))}$ and $|p|_{H^{1+(\mathfrak{d}(e))}}=|p|_{H^{1}(\mathfrak{d}(e))}+h_{e}|p|_{H^{2}(\mathfrak{d}(e))} \cdot$

Proof. The bound on the diffusion-related terms derived in Theorem 4.4 still holds. For the advectionrelated terms, there are two adaptations from the proof of Theorem 3.4. The first one is that we consider $\mathbf{R}_{\mathcal{V}}(p)$ in lieu of $\widehat{R}_{\mathcal{V}}(p)$ since we are now bounding the error $\left(\mathbf{p}-\mathbf{R}_{\mathcal{V}}(p)\right)$. The approximation property (3.21), which is now applied in the diamonds around edges, is then replaced by

$$
\left\|q-\left(\mathrm{R}_{\mathcal{V}}(q)\right)_{v}\right\|_{L^{2}(\mathfrak{d}(e))}+h_{e}^{\frac{1}{2}}\left\|q-\left(\mathrm{R}_{\mathcal{V}}(q)\right)_{v}\right\|_{L^{2}(\tilde{f}(e))} \lesssim h_{e}|q|_{H^{1+}(\mathfrak{d}(e))},
$$

for all $q \in H^{2}(\Omega)$, all $e \in \mathrm{E}$, and all $v \in \mathrm{V}_{e}$. The second adaptation is related to the change in the $\|\cdot\|_{\text {upw }, \beta}$ semi-norm owing to the use of Péclet-based upwinding. We bound again the three terms in the right-hand side of (3.19) for all $\mathrm{q} \in \mathcal{V}$ such that $\|\mathrm{q}\|_{\text {ad, } \mathcal{V}}=1$. For the first term, we readily infer that

$$
\left|\llbracket \mathbf{q},\left\lfloor\mathrm{H}_{-\nabla \cdot \boldsymbol{\beta}}^{\nu \tilde{\mathcal{C}}}, \mathrm{R}\right\rceil(p) \rrbracket_{\mathcal{\nu} \tilde{\mathcal{C}}}\right| \lesssim\left(\tau^{-\frac{1}{2}}\|\mathbf{q}\|_{2, \mathcal{V}}\right)\left(\sum_{e \in \mathrm{E}} \tau|\nabla \cdot \boldsymbol{\beta}|_{L^{\infty}(\mathfrak{d}(e))}^{2} h_{e}^{2}|p|_{H^{1+(\mathfrak{d}(e))}}^{2}\right)^{\frac{1}{2}}
$$

Consider now the second term. Let $\mathrm{E}_{>1}:=\left\{e \in \mathrm{E}|| \mathrm{Pe} e_{e} \mid>1\right\}$ and $\mathrm{E}_{\leq 1}:=\left\{e \in \mathrm{E}|| \mathrm{Pe}_{e} \mid \leq 1\right\}$. The summation in the right-hand side of $(3.22)$ is split as $\sum_{e \in \mathrm{E}_{>1}}(\cdot)+\sum_{e \in \mathrm{E}_{\leq 1}}(\cdot)$. Proceeding as in the proof of Theorem 3.4, we infer that

$$
\sum_{e \in \mathrm{E}_{>1}}(\cdot) \lesssim\left(\sum_{e \in \mathrm{E}_{>1}} \llbracket \mathrm{q} \rrbracket_{e}^{2} \int_{\tilde{f}(e)}\left|\boldsymbol{\beta} \cdot \boldsymbol{n}_{\tilde{f}(e)}\right| d \tilde{f}\right)^{\frac{1}{2}}\left(\sum_{e \in \mathrm{E}_{>1}}|\boldsymbol{\beta} \cdot \boldsymbol{n}|_{\sharp, e} h_{e}|p|_{H^{1+}(\mathfrak{d}(e))}^{2}\right)^{\frac{1}{2}} .
$$

For all $e \in \mathrm{E}_{>1}$, property $(\Theta 2)$ implies that $\Lambda_{e} \beta_{e} \geq \alpha\left|\beta_{e}\right|$. Then, still proceeding as in the proof of Theorem 3.4, we infer that

$$
\sum_{e \in \mathrm{E}_{>1}}(\cdot) \lesssim\left(\|\mathbf{q}\|_{\text {upw }, \boldsymbol{\beta}}+\tau^{-\frac{1}{2}}\|\mathbf{q}\|_{2, \mathcal{V}}\right)\left(\sum_{e \in \mathrm{E}_{>1}}|\boldsymbol{\beta} \cdot \boldsymbol{n}|_{\sharp, e} h_{e}|p|_{H^{1+(\mathfrak{d}(e))}}^{2}\right)^{\frac{1}{2}} .
$$

Furthermore, we observe that

$$
\sum_{e \in \mathrm{E}_{\leq 1}}(\cdot) \lesssim\left(\sum_{e \in \mathrm{E}_{\leq 1}} \llbracket \mathrm{q} \rrbracket_{e}^{2} h_{e} \lambda_{e}\right)^{\frac{1}{2}}\left(\sum_{e \in \mathrm{E}_{\leq 1}} h_{e}^{-1} \lambda_{e}^{-1}|\boldsymbol{\beta} \cdot \boldsymbol{n}|_{\sharp, e}\left|\beta_{e}\right| h_{e}|p|_{H^{1+(\mathfrak{d}(e))}}^{2}\right)^{\frac{1}{2}} .
$$

Owing to mesh regularity, the definition of $\lambda_{e}$, and (H1), we infer that the first factor in the right-hand side is bounded by $\|\operatorname{GRAD}(\mathbf{q})\|_{\boldsymbol{\lambda}}$, while the second factor is bounded by $\left(\sum_{e \in \mathrm{E}}|\boldsymbol{\beta} \cdot \boldsymbol{n}|_{\sharp, e} h_{e} \mathrm{Pe}_{e}|p|_{H^{1+}(\mathfrak{d}(e))}^{2}\right)^{\frac{1}{2}}$ since 
$\lambda_{e}^{-1}|\tilde{f}(e)|^{-1}\left|\beta_{e}\right| h_{e}=\left|\mathrm{Pe}_{e}\right| \leq 1$ and $|\tilde{f}(e)| \lesssim h_{e}^{2}$. Collecting the bounds on $\sum_{e \in \mathrm{E}_{>1}}$ and $\sum_{e \in \mathrm{E}_{\leq 1}}$ leads to

$$
\left.\mid \llbracket \operatorname{GRAD}(\mathbf{q}),\lfloor\rfloor_{\boldsymbol{\beta}}^{\mathcal{\mathcal { F }}}, \widehat{\mathrm{R}}\right\rceil(p) \rrbracket_{\mathcal{E} \tilde{\mathcal{F}}} \mid \lesssim\|\mathbf{q}\|_{\text {ad, } \mathcal{V}}\left(\sum_{e \in \mathrm{E}}|\boldsymbol{\beta} \cdot \boldsymbol{n}|_{\sharp, e} h_{e} \min \left(1, \mathrm{Pe} e_{e}\right)|p|_{H^{1+(\mathfrak{d}(e))}}^{2}\right)^{\frac{1}{2}}
$$

Finally, the boundary term is bounded as before.

Remark 4.1 (Limit regimes). In the advection-dominant regime with $\left|\mathrm{Pe}_{e}\right| \geq 1$ for all $e \in \mathrm{E}$, the error bound (4.22) behaves as $h^{1 / 2}$ (see Theorem 3.4), while, in the diffusion-dominant regime with $\left|\mathrm{Pe}_{e}\right| \leq h_{e}$ for all $e \in \mathrm{E}$, it behaves as $h$ (see Theorem 4.4). The case where $h_{e} \leq \mathrm{Pe}_{e} \leq 1$ corresponds to transition regimes and intermediate orders of convergence.

Remark 4.2 (Boundary term). It is also possible to modify the discrete boundary Hodge operator so as to enforce the boundary condition using a Péclet-based upwinding; details are omitted for brevity.

\section{Divergence-free advection}

In this section, we extend the analysis to the case of a divergence-free velocity field $\boldsymbol{\beta}$ under assumption $(\boldsymbol{\beta 2})$; recall that this assumption provides a real number $\tau>0$ and a function $\zeta \in W^{1, \infty}(\Omega)$ such that $\zeta \geq 1$ a.e. in $\Omega$. The advection-related stability norm $\|\cdot\|_{a, \mathcal{V}}$ is still defined by (3.14) (where now $\tau$ results from $(\boldsymbol{\beta 2})$ ). The only relevant change in the analysis is that stability (and well-posedness) is now achieved by means of an inf-sup condition instead of a coercivity argument. Since consistency and boundedness hold in the same form as before, inf-sup stability suffices to establish the error upper bounds, so that the convergence rates derived in Theorem 3.4 for pure advection and in Theorem 4.7 for advection-diffusion still hold. In what follows, we consider the non-dimensional numbers $\omega_{\beta}=L_{\zeta}^{2}|\beta|_{\sharp} h \tau$ and $\omega_{\lambda}=L_{\zeta}^{2} \lambda_{\sharp} \tau$, with $L_{\zeta}$ the Lipschitz constant of $\zeta,|\boldsymbol{\beta}|_{\sharp}:=\|\boldsymbol{\beta}\|_{\boldsymbol{L}^{\infty}(\Omega)}$, and $\lambda_{\sharp}:=\max _{c \in \mathrm{C}} \lambda_{\sharp, c}$.

\subsection{Pure advection}

Along with (I1)-(I2), we introduce a third property for the discrete contraction operators:

(I3) [Multiplication by $\zeta$ ] There are $c_{1}, c_{2}, c_{3}$, uniform with respect to the mesh-size and the functions $\boldsymbol{\beta}$ and $\zeta$, such that the following holds for all $\mathrm{q} \in \mathcal{V}$ :

$$
\begin{aligned}
\|\zeta \mathrm{q}\|_{\text {upw }, \boldsymbol{\beta}}^{2}+\|\zeta \mathrm{q}\|_{|\boldsymbol{\beta} \cdot \boldsymbol{n}|}^{2} & \leq c_{1} \zeta_{\sharp}^{2}\left(\|\mathrm{q}\|_{\text {upw }, \boldsymbol{\beta}}^{2}+\|\mathrm{q}\|_{|\boldsymbol{\beta} \cdot \boldsymbol{n}|}^{2}\right)+c_{2} \omega_{\beta} \tau^{-1}\|\mathrm{q}\|_{2, \mathcal{V}}^{2}, \\
\mathrm{~A}_{\boldsymbol{\beta}}(\mathbf{q}, \zeta \mathrm{q}) & \geq \frac{1}{4}\|\mathrm{q}\|_{\mathrm{a}, \mathcal{V}}^{2}-c_{3} \omega_{\beta} \mathrm{A}_{\boldsymbol{\beta}}(\mathbf{q}, \mathbf{q}),
\end{aligned}
$$

with $\zeta_{\sharp}:=\|\zeta\|_{L^{\infty}(\Omega)}$ and $\zeta \mathbf{q} \in \mathcal{V}$ such that $(\zeta \mathbf{q})_{v}:=\zeta(v) \mathbf{q}_{v}$ for all $v \in \mathrm{V}$.

Lemma 5.1 (Inf-sup stability). Under hypotheses (及2), (I1)-(I2)-(I3), and $\left(\boldsymbol{H}^{\partial}\right)$, the following holds:

$$
\varrho\|q\|_{a, \mathcal{V}} \leq \sup _{r \in \mathcal{V}} \frac{A_{\beta}(q, r)}{\|r\|_{a, \mathcal{V}}}, \quad \forall q \in \mathcal{V}
$$

with $\varrho=\frac{1}{4}\left(\max \left(\zeta_{\sharp}^{2}+c_{2} \omega_{\beta}, c_{1} \zeta_{\sharp}^{2}\right)^{\frac{1}{2}}+c_{3} \omega_{\beta}\right)^{-1}$. 
Proof. We take $r=\zeta q+c_{3} \omega_{\beta} q \in \mathcal{V}$. Owing to the triangle inequality, (5.1a), and the obvious bound $\|\zeta \mathrm{q}\|_{2, \mathcal{V}} \leq \zeta_{\sharp}\|\mathrm{q}\|_{2, \mathcal{V}}$, we infer that

$$
\|\mathbf{r}\|_{\mathrm{a}, \mathcal{V}} \leq\|\zeta \mathrm{q}\|_{\mathrm{a}, \mathcal{V}}+c_{3} \omega_{\beta}\|\mathbf{q}\|_{\mathrm{a}, \mathcal{V}} \leq\left(\max \left(\zeta_{\sharp}^{2}+c_{2} \omega_{\beta}, c_{1} \zeta_{\sharp}^{2}\right)^{\frac{1}{2}}+c_{3} \omega_{\beta}\right)\|\mathbf{q}\|_{\mathrm{a}, \mathcal{V}} .
$$

Moreover, owing to (5.1b), we infer that

$$
\mathrm{A}_{\beta}(\mathrm{q}, \mathrm{r})=\mathrm{A}_{\beta}(\mathrm{q}, \zeta \mathrm{q})+c_{3} \omega_{\beta} \mathrm{A}_{\beta}(\mathrm{q}, \mathrm{q}) \geq \frac{1}{4}\|\mathrm{q}\|_{a, \mathcal{V}}^{2},
$$

whence we infer (5.2).

Remark 5.1 (Factor $\left.\omega_{\beta}\right)$. An upper bound on $\omega_{\beta}$ yields a lower bound on $\varrho$. A simple upper bound is to replace $h$ by a global length scale associated with $\Omega$ (i.e., $h$ can be replaced by a global length scale in (5.1a) and (5.1b)). A sharper bound is $\omega_{\beta} \leq L_{\zeta}|\boldsymbol{\beta}|_{\sharp} \tau$ under the mild assumption $L_{\zeta} h \leq 1$ (meaning that $h$ resolves the scale of spatial variations of $\zeta$ ).

We now verify property (I3) in the context of the CDO scheme with upwinding studied in Section 3.2.

Lemma 5.2 ((I3) with upwinding). Assume $(\boldsymbol{\beta} 2)$ and $(\Lambda 1)$. Let the discrete contraction and surface Hodge operators be given by (3.10)-(3.11)-(3.12). Then, (I3) holds.

Proof. To prove property (5.1a), we observe that, for all $\mathrm{q} \in \mathcal{V}$, since $\Lambda_{e} \beta_{e} \geq 0$ for all $e \in \mathrm{E}$,

$$
\|\zeta \mathrm{q}\|_{\text {upw }, \beta}^{2}=\sum_{e \in \mathrm{E}} \llbracket \zeta \mathrm{q} \rrbracket_{e}^{2} \Lambda_{e} \beta_{e} \leq 2 \sum_{e \in \mathrm{E}}\left(\{\zeta \zeta\}_{e}^{2} \llbracket \mathrm{q} \rrbracket_{e}^{2}+\left\{\{\mathrm{q}\}_{e}^{2} \llbracket \zeta \rrbracket_{e}^{2}\right) \Lambda_{e} \beta_{e}\right.
$$

where $\{\zeta \zeta\}_{e}=\frac{1}{2} \sum_{v \in \mathrm{V}_{e}} \zeta(v), \llbracket \zeta \rrbracket_{e}=\sum_{v \in \mathrm{V}_{e}} \iota_{\tilde{f}(e), \tilde{c}(v)} \zeta(v),\left\{\{\mathbf{q}\}_{e}=\frac{1}{2} \sum_{v \in \mathrm{V}_{e}} \mathbf{q} v\right.$, and $\llbracket \mathbf{q} \rrbracket_{e}$ is defined in Lemma 3.1. Since $2\{\zeta \zeta\}_{e}^{2} \leq 2 \zeta_{\sharp}^{2}$ and $\llbracket \zeta \rrbracket_{e}^{2} \leq\left(L_{\zeta} h_{e}\right)^{2}$, we infer that

$$
\|\zeta \mathrm{q}\|_{\text {upw }, \beta}^{2} \leq 2 \zeta_{\sharp}^{2}\|\mathrm{q}\|_{\text {upw }, \beta}^{2}+2 \sum_{e \in \mathrm{E}} L_{\zeta}^{2} h_{e}^{2}\left\{\{\mathrm{q}\}_{e}^{2} \Lambda_{e} \beta_{e},\right.
$$

and we conclude using $0 \leq \Lambda_{e} \beta_{e} \leq|\boldsymbol{\beta}|_{\sharp}|\tilde{f}(e)|,\left\{\{q\}_{e}^{2} \leq \frac{1}{2} \sum_{v \in \mathrm{V}_{e}} \mathrm{q}_{v}^{2}\right.$, and mesh regularity. Since, owing to (3.12), $\|\zeta \mathrm{q}\|_{|\boldsymbol{\beta} \cdot \boldsymbol{n}|}^{2} \leq \zeta_{\sharp}^{2}\|\mathrm{q}\|_{|\boldsymbol{\beta} \cdot \boldsymbol{n}|}^{2}$, this completes the proof of (5.1a).

Proof of $(5.1 \mathrm{~b})$. The idea of the proof consists of writing $\mathrm{A}_{\beta}(\mathrm{q}, \zeta \mathrm{q})$ in the form $\mathrm{A}_{\zeta \beta}(\mathrm{q}, \mathrm{q})$ plus a perturbation which can be bounded by the variations of $\zeta$. A straightforward computation proceeding as in the proof of Lemma 3.1 shows that $\mathrm{A}_{\boldsymbol{\beta}}(\mathbf{q}, \zeta \mathrm{q})=T_{1}+T_{2}+T_{3}$ with

$$
\begin{aligned}
T_{1} & =\sum_{v \in \mathrm{V}} \sum_{e \in \mathrm{E}_{v}} \frac{1}{2} \mathrm{q}_{v}^{2} \int_{\tilde{f}(e)}(-\zeta \boldsymbol{\beta}) \cdot \boldsymbol{n}_{\tilde{f}(e)} d \tilde{f}+\sum_{e \in \mathrm{E}} \frac{1}{2} \llbracket \mathrm{q} \rrbracket_{e}^{2} \zeta_{e} \Lambda_{e} \beta_{e}+\sum_{v \in \mathrm{V}^{\partial}} \mathrm{q}_{v}^{2} \int_{\tilde{f}^{\partial}(v)} \zeta(\boldsymbol{\beta} \cdot \boldsymbol{n})^{-} d \tilde{f}, \\
T_{2} & =\sum_{v \in \mathrm{V}} \sum_{e \in \mathrm{E}_{v}} \frac{1}{2} \mathrm{q}_{v} \mathrm{~g}_{e} \int_{\tilde{f}(e)}(\zeta(v)-\zeta) \boldsymbol{\beta} \cdot \boldsymbol{n}_{\tilde{f}(e)} d \tilde{f}+\sum_{v \in \mathrm{V}} \sum_{e \in \mathrm{E}_{v}} \frac{1}{2} \mathrm{q}_{v} \mathrm{~g}_{e}\left(\zeta_{e}-\zeta(v)\right) \Lambda_{v e} \beta_{e}, \\
T_{3} & =\sum_{v \in \mathrm{V}^{\partial}} \mathrm{q}_{v}^{2} \int_{\tilde{f}^{\partial}(v)}(\zeta(v)-\zeta)(\boldsymbol{\beta} \cdot \boldsymbol{n})^{-} d \tilde{f},
\end{aligned}
$$

with $\mathrm{g}=\operatorname{GRAD}(\mathrm{q})$ and $\zeta_{e}$ the mean-value of $\zeta$ in $e$. Since $\zeta \geq 1$, still proceeding as in the proof of Lemma 3.1 and using now $(\boldsymbol{\beta 2})$ leads to the bound $T_{1} \geq \frac{1}{2}\|\mathrm{q}\|_{\mathrm{a}, \mathcal{V}}^{2}$. Furthermore, using Cauchy-Schwarz inequalities, $(\Lambda 2)$, and mesh regularity, we obtain $\left|T_{2}\right| \lesssim \omega_{\beta}\|\mathbf{q}\|_{\text {upw, } \beta}\left(\tau^{-\frac{1}{2}}\|\mathbf{q}\|_{2, \mathcal{V}}\right)$. Proceeding similarly leads 
to $\left|T_{3}\right| \lesssim \omega_{\beta}\|\mathbf{q}\|_{|\boldsymbol{\beta} \cdot \boldsymbol{n}|}\left(\tau^{-\frac{1}{2}}\|\mathbf{q}\|_{2, \mathcal{V}}\right)$. Since $\mathrm{A}_{\boldsymbol{\beta}}(\mathbf{q}, \mathbf{q}) \geq \frac{1}{2}\left(\|\mathbf{q}\|_{\text {upw }, \boldsymbol{\beta}}^{2}+\|\mathbf{q}\|_{|\boldsymbol{\beta} \cdot \boldsymbol{n}|}^{2}\right)$ owing to (3.16), we infer that

$$
\left|T_{2}\right|+\left|T_{3}\right| \lesssim \omega_{\beta} \mathrm{A}_{\beta}(\mathbf{q}, \mathbf{q})^{\frac{1}{2}}\left(\tau^{-\frac{1}{2}}\|\mathbf{q}\|_{2, \mathcal{V}}\right)
$$

and the conclusion follows using Young's inequality.

Remark 5.2 (Conservative advection). Using the conservative form of the advective derivative is also possible under assumption $(\boldsymbol{\beta 2})$. The above proofs are adapted by considering the function $\zeta^{\prime}=1+\|\zeta\|_{L^{\infty}(\Omega)}-\zeta$ which is bounded by $\|\zeta\|_{L^{\infty}(\Omega)}$ and satisfies $\zeta^{\prime} \geq 1$ and $\nabla \cdot\left(\zeta^{\prime} \boldsymbol{\beta}\right) \geq \tau^{-1}$ a.e. in $\Omega$.

\subsection{Advection-diffusion}

As in Section 4.3, we consider the Péclet-dependent upwinding parameters $\Lambda_{v e}=\Theta\left(\iota_{\tilde{f}(e), \tilde{c}(v)} \mathrm{Pe}_{e}\right)$ under assumptions $(\Theta 1)-(\Theta 2)$. Recall that $(\Theta 1)$ implies $(\Lambda 1)$.

Lemma 5.3 (Inf-sup stability). Assume (及2). Let the discrete contraction and surface Hodge operators be given by (3.10)-(3.11)-(3.12) with Péclet-dependent upwinding parameters under assumption ( $(\Theta 1)$. Assume (H1) and (N1) for the diffusion-related terms. Then, provided $\eta_{0} \geq \frac{1+2 c_{\mathrm{N}}^{2}\left(\zeta_{\sharp}+c_{4}^{2}\right)}{7\left(1+c_{4}\right)}$ with $c_{4}:=$ $\max \left(c_{3} \omega_{\beta}, 2 c_{5} \omega_{\lambda}^{2}\right), c_{5}:=\left(2 c_{\mathrm{H}}^{-1} N_{\mathrm{V}, \mathrm{E}}\right)^{\frac{1}{2}}, N_{\mathrm{V}, \mathrm{E}}$ being the maximum number of edges touching a mesh vertex, the following holds:

$$
\varrho\|q\|_{a d, \mathcal{V}} \leq \sup _{r \in \mathcal{V}} \frac{A_{\beta, \lambda}(q, r)}{\|r\|_{a d}, \mathcal{V}}, \quad \forall q \in \mathcal{V}
$$

with $\varrho=\frac{1}{8}\left(\max \left(\zeta_{\sharp}^{2}+c_{2} \omega_{\beta}+2 c_{5}^{2} \omega_{\lambda}, c_{1} \zeta_{\sharp}^{2}, 2 \zeta_{\sharp}^{2}\right)^{\frac{1}{2}}+c_{4}\right)^{-1}$.

Proof. Set $r:=\zeta q+c_{4} q$. Since $(\Lambda 1)$ holds, we infer from Lemma 5.2 that $(\mathbf{I} 3)$ holds. Moreover, since $c_{4} \geq c_{3} \omega_{\beta}$ and $\mathrm{A}_{\boldsymbol{\beta}}(\mathbf{q}, \mathbf{q}) \geq 0$, Lemma 5.1 implies that

$$
\mathrm{A}_{\beta}(\mathrm{q}, \mathrm{r}) \geq \mathrm{A}_{\beta}\left(\mathrm{q}, \zeta \mathrm{q}+c_{3} \omega_{\beta} \mathrm{q}\right) \geq \frac{1}{4}\|\boldsymbol{q}\|_{\mathrm{a}, \mathcal{V}}^{2}
$$

Moreover, owing to (7.2) and to Lemma 5.4 below, we infer that

$$
\mathrm{A}_{\boldsymbol{\lambda}}(\mathrm{q}, \mathrm{r}) \geq\left(1+c_{4}\right)\|\mathrm{g}\|_{\lambda}^{2}-c_{\mathrm{N}}\left(\zeta_{\sharp}+c_{4}\right)\|\mathrm{g}\|\left\|_{\lambda}\right\| \mathrm{q}\left\|_{\lambda / h}-c_{5} \omega_{\lambda}^{\frac{1}{2}}\right\| \mathrm{g}\left\|_{\boldsymbol{\lambda}} \tau^{-\frac{1}{2}}\right\| \mathrm{q}\left\|_{2, \mathcal{V}}+\eta_{0}\left(1+c_{4}\right)\right\| \mathrm{q} \|_{\lambda / h}^{2},
$$

where we have set $g=\operatorname{GRAD}(q)$. Using Young's inequality for the third term on the right-hand side yields

$$
\mathrm{A}_{\boldsymbol{\lambda}}(\mathbf{q}, \mathrm{r}) \geq\|\mathrm{g}\|_{\lambda}^{2}-c_{\mathrm{N}}\left(\zeta_{\sharp}+c_{4}\right)\|\mathrm{g}\|_{\lambda}\|\mathbf{q}\|_{\lambda / h}+\eta_{0}\left(1+c_{4}\right)\|\mathbf{q}\|_{\lambda / h}^{2}-\frac{1}{8} \tau^{-1}\|\mathbf{q}\|_{2, \mathcal{V}}^{2},
$$

since $c_{4} \geq 2 c_{5} \omega_{\lambda}^{2}$. Using the same quadratic identity as in Lemma 4.5, this time with $\gamma=\frac{1}{2} c_{\mathrm{N}}\left(\zeta_{\sharp}+c_{4}\right)$ and $\delta=\eta_{0}\left(1+c_{4}\right)$, and observing that the choice $\eta_{0} \geq \frac{1+2 c_{N}^{2}\left(\zeta_{\sharp}+c_{4}^{2}\right)}{7\left(1+c_{4}\right)}$ implies $\delta \geq \frac{1}{7}+\frac{8}{7} \gamma^{2}$ so that $\frac{\delta-\gamma^{2}}{1+\delta} \geq \frac{1}{8}$, we infer that

$$
\mathrm{A}_{\lambda}(\mathrm{q}, \mathrm{r}) \geq \frac{1}{8}\|\mathrm{q}\|_{\mathrm{d}, \mathcal{V}}^{2}-\frac{1}{8} \tau^{-1}\|\mathrm{q}\|_{2, \mathcal{V}}^{2}
$$

Combining this bound with (5.4) yields $A_{\beta, \lambda}(\mathrm{q}, \mathrm{r}) \geq \frac{1}{8}\|\mathrm{q}\|_{\text {ad, } \mathcal{V}}^{2}$. We conclude using $\|\mathrm{r}\|_{\text {ad }, \mathcal{V}} \leq\|\zeta \mathrm{q}\|_{\text {ad, } \mathcal{V}}+$ $c_{4}\|\mathbf{q}\|_{\text {ad, } \mathcal{V}}$ and $\|\zeta \mathbf{q}\|_{\text {ad, } \mathcal{V}} \leq \max \left(\zeta_{\sharp}^{2}+c_{2} \omega_{\beta}+2 c_{5}^{2} \omega_{\lambda}, c_{1} \zeta_{\sharp}^{2}, 2 \zeta_{\sharp}^{2}\right)^{\frac{1}{2}}\|\mathbf{q}\|_{\text {ad, }, \mathcal{V}}$.

Remark $5.3\left(\eta_{0}\right)$. The lower bound for $\eta_{0}$ obtained in Lemma 5.3 slightly differs, up to a numerical factor, from that obtained in Lemma 4.2 for zero advection; the reason is that both proofs have not been optimized regarding the lower bound in the quadratic identity. 
Lemma 5.4 (Multiplication by $\zeta$ ). Assume that (H1) and (N1) hold. The following holds for all $\mathrm{q} \in \mathcal{V}$ with $\mathrm{g}=\operatorname{GRAD}(\mathbf{q})$ :

$$
\begin{aligned}
\|\zeta \mathrm{q}\|_{\mathbf{d}, \mathcal{V}}^{2} & \leq 2 \zeta_{\sharp}^{2}\|\mathbf{q}\|_{\mathbf{d}, \mathcal{V}}^{2}+2 c_{5}^{2} \omega_{\lambda} \tau^{-1}\|\mathbf{q}\|_{2, \mathcal{V}}^{2}, \\
A_{\lambda}(\mathbf{q}, \zeta \mathbf{q}) & \geq\|\mathrm{g}\|_{\lambda}^{2}+\eta_{0}\left\|\mathbf{q}^{\partial}\right\|_{\lambda / h}^{2}-c_{N} \zeta_{\sharp}\|\mathrm{g}\|_{\lambda}\left\|\mathbf{q}^{\partial}\right\|_{\lambda / h}-c_{5} \omega_{\lambda}^{\frac{1}{2}}\|\mathrm{~g}\|_{\lambda}\left(\tau^{-\frac{1}{2}}\|\mathrm{q}\|_{2, \mathcal{V}}\right) .
\end{aligned}
$$

Proof. Proof of (5.5a). The definition of $\widehat{\boldsymbol{H}}_{\lambda / h}^{\partial}$ implies that $\left\|\zeta \mathbf{q}^{\partial}\right\|_{\lambda / h}^{2}=\llbracket \zeta \mathbf{q}^{\partial}, \widehat{\boldsymbol{H}}_{\lambda / h}^{\partial}\left(\zeta \mathbf{q}^{\partial}\right) \rrbracket_{(\mathcal{\nu} \tilde{\mathcal{F}})^{\partial}} \leq \zeta_{\sharp}^{2}\left\|\mathbf{q}^{\partial}\right\|_{\lambda / h}^{2}$. Furthermore, owing to the cell-wise assembly of the discrete Hodge operator $\mathrm{H}_{\lambda}^{\mathcal{E}}$ and using the triangle inequality, we infer that

$$
\begin{aligned}
\|\operatorname{GRAD}(\zeta \mathbf{q})\|_{\lambda}^{2} & =\sum_{c \in \mathrm{C}} \llbracket \operatorname{GRAD}_{c}(\zeta \mathbf{q}), \mathrm{H}_{\lambda}^{\left((\mathcal{\mathcal { F }})_{c}\right.} \cdot \operatorname{GRAD}_{c}(\zeta \mathbf{q}) \rrbracket_{(\mathcal{\varepsilon} \tilde{\mathcal{F}})_{c}} \\
& =\sum_{c \in \mathrm{C}} 2 \zeta_{c}^{2} \llbracket \operatorname{GRAD}_{c}(\mathbf{q}), \mathrm{H}_{\lambda}^{(\tilde{\mathcal{F}} \tilde{\mathcal{F}})} \cdot \operatorname{GRAD}_{c}(\mathbf{q}) \rrbracket_{(\mathcal{\varepsilon} \tilde{\mathcal{F}})_{c}}+\sum_{c \in \mathrm{C}} 2 \delta_{c}(\mathbf{q})^{2} \\
& \leq 2 \zeta_{\sharp}^{2}\|\operatorname{GRAD}(\mathbf{q})\|_{\lambda}^{2}+\sum_{c \in \mathrm{C}} 2 \delta_{c}(\mathbf{q})^{2},
\end{aligned}
$$

where $\zeta_{c}$ is the value of $\zeta$ at the barycenter of $c$ and $\delta_{c}(\mathrm{q}):=\left\|\operatorname{GRAD}_{c}(\mathrm{w})\right\|_{\boldsymbol{\lambda}, c}$ and $\mathrm{w}:=\left(\zeta-\zeta_{c}\right) \mathrm{q}$. The upper bound in (H1), the definition of $\mathrm{GRAD}_{c}$ and that of the $\|\cdot\|_{2, \mathcal{E}_{c}}$-norm yield

$$
\begin{aligned}
\delta_{c}(\mathbf{q})^{2} & \leq c_{\mathrm{H}}^{-1} \lambda_{\sharp, c} h_{c} \sum_{e \in \mathrm{E}_{c}}\left(\sum_{v \in \mathrm{V}_{e}} \iota_{v, e}\left(\zeta(v)-\zeta_{c}\right) \mathrm{q}_{v}\right)^{2} \\
& \leq 2 c_{\mathrm{H}}^{-1} \lambda_{\sharp, c} L_{\zeta}^{2} h_{c}^{3} \sum_{e \in \mathrm{E}_{c}} \sum_{v \in \mathrm{V}_{e}} \mathrm{q}_{v}^{2} \leq 2 c_{\mathrm{H}}^{-1} N_{\mathrm{V}, \mathrm{E}} \lambda_{\sharp, c} L_{\zeta}^{2}\|\mathrm{q}\|_{2, \mathcal{V}_{c}}^{2} .
\end{aligned}
$$

Combining the above bounds leads to (5.5a).

Proof of (5.5b). Using (N1), (7.1), and $\zeta \geq 1$, we infer that

$$
\begin{aligned}
\mathrm{A}_{\boldsymbol{\lambda}}(\mathbf{q}, \zeta \mathbf{q}) & =\llbracket \operatorname{GRAD}(\zeta \mathbf{q}), \mathrm{H}_{\lambda}^{\mathcal{\mathcal { F }}} \cdot \operatorname{GRAD}(\mathbf{q}) \rrbracket_{\mathcal{E} \tilde{\mathcal{F}}}-\llbracket(\zeta \mathbf{q})^{\partial}, \mathrm{N}_{\lambda}^{\partial} \cdot \operatorname{GRAD}(\mathbf{q}) \rrbracket_{(\nu \tilde{\mathcal{F}})^{\partial}}+\eta_{0} \llbracket(\zeta \mathbf{q})^{\partial}, \widehat{H}_{\lambda / h}^{\partial}\left(\mathbf{q}^{\partial}\right) \rrbracket_{(\nu \tilde{\mathcal{F}})} \\
& \geq \llbracket \operatorname{GRAD}(\zeta \mathbf{q}), \mathrm{H}_{\lambda}^{\mathcal{E} \tilde{\mathcal{F}}} \cdot \operatorname{GRAD}(\mathbf{q}) \rrbracket_{\mathcal{E} \tilde{\mathcal{F}}}-c_{\mathrm{N}}\|\operatorname{GRAD}(\mathbf{q})\|_{\lambda}\left\|(\zeta \mathbf{q})^{\partial}\right\|_{\lambda / h}+\eta_{0}\left\|\mathbf{q}^{\partial}\right\|_{\lambda / h}^{2} \\
& \geq \llbracket \operatorname{GRAD}(\zeta \mathbf{q}), \mathrm{H}_{\lambda}^{\mathcal{E} \tilde{\mathcal{F}}} \cdot \operatorname{GRAD}(\mathbf{q}) \rrbracket_{\mathcal{E} \tilde{\mathcal{F}}}-c_{\mathbf{N}} \zeta_{\sharp}\|\operatorname{GRAD}(\mathbf{q})\|_{\lambda}\left\|\mathbf{q}^{\partial}\right\|_{\lambda / h}+\eta_{0}\left\|\mathbf{q}^{\partial}\right\|_{\lambda / h}^{2} .
\end{aligned}
$$

Moreover, owing to the cell-wise assembly of the discrete Hodge operator $\mathrm{H}_{\lambda}^{\mathcal{E}} \tilde{\mathcal{F}}$ and proceeding as above, we infer that

$$
\begin{aligned}
\llbracket \operatorname{GRAD}(\zeta \mathrm{q}), \mathrm{H}_{\lambda}^{\mathcal{E \mathcal { F }}} \cdot \operatorname{GRAD}(\mathrm{q}) \rrbracket_{\mathcal{E} \tilde{\mathcal{F}}} & =\sum_{c \in \mathrm{C}} \llbracket \operatorname{GRAD}_{c}(\zeta \mathrm{q}), \mathrm{H}_{\lambda}^{(\mathcal{\mathcal { F }})_{c}} \cdot \operatorname{GRAD}_{c}(\mathbf{q}) \rrbracket_{(\mathcal{E} \tilde{\mathcal{F}})_{c}} \\
& \geq\|\operatorname{GRAD}(\mathbf{q})\|_{\lambda}^{2}+\sum_{c \in \mathrm{C}} \llbracket \operatorname{GRAD}_{c}(\mathrm{w}), \mathrm{H}_{\lambda}^{(\mathcal{( \varepsilon \mathcal { F }})_{c}} \cdot \operatorname{GRAD}_{c}(\mathbf{q}) \rrbracket_{(\mathcal{E} \tilde{\mathcal{F}})_{c}}
\end{aligned}
$$

Since $\mathrm{H}_{\lambda}^{(\mathcal{\varepsilon} \tilde{\mathcal{F}})_{c}}$ is self-adjoint and monotone, we infer that

$$
\mid \llbracket\left[\operatorname{GRAD}_{c}(\mathrm{w}), \mathrm{H}_{\lambda}^{(\mathcal{E} \tilde{\mathcal{F}})_{c}} \cdot \operatorname{GRAD}_{c}(\mathbf{q}) \rrbracket_{(\mathcal{\varepsilon} \tilde{\mathcal{F}})_{c}} \mid \leq \delta_{c}(\mathbf{q})\left\|\operatorname{GRAD}_{c}(\mathbf{q})\right\|_{\lambda, c}\right.
$$


so that

$$
\llbracket \operatorname{GRAD}(\zeta \mathbf{q}), \mathrm{H}_{\lambda}^{\mathcal{E} \tilde{\mathcal{F}}} \cdot \operatorname{GRAD}(\mathbf{q}) \rrbracket_{\mathcal{E} \tilde{\mathcal{F}}} \geq\|\operatorname{GRAD}(\mathbf{q})\|_{\lambda}^{2}-\left(\sum_{c \in \mathrm{C}} \delta_{c}(\mathbf{q})^{2}\right)^{\frac{1}{2}}\|\operatorname{GRAD}(\mathbf{q})\|_{\lambda} .
$$

Using the above bound on $\delta_{c}(\mathrm{q})$ yields $(5.5 \mathrm{~b})$.

\section{$6 \quad$ Numerical results}

In this section, we investigate numerically CDO advection-diffusion schemes on four families of successivelyrefined, polyhedral meshes of the unit cube $\Omega=(0,1)^{3}$; see Figure 5 for an example of mesh within each family. These mesh families have been proposed in the FVCA benchmark [24], see also [7].

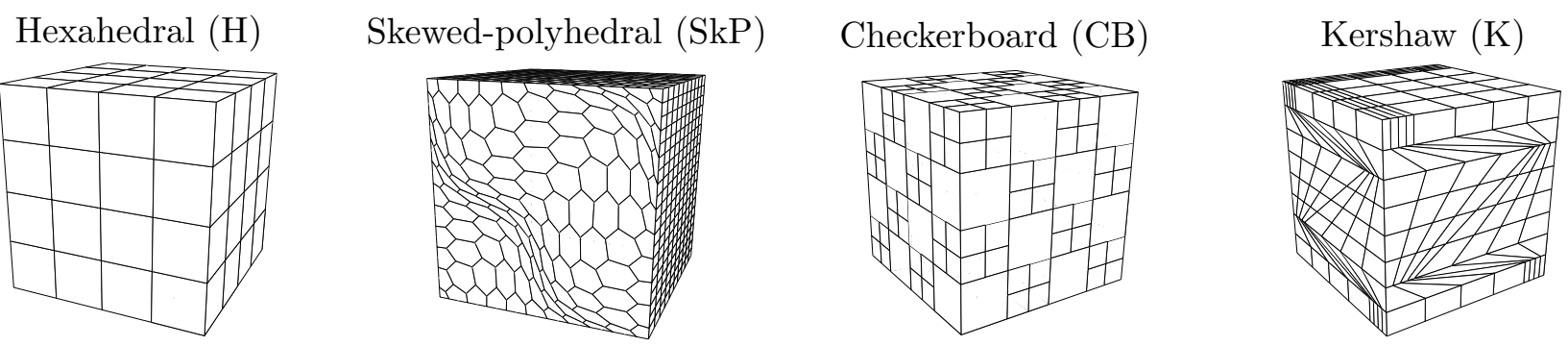

Figure 5: Polyhedral meshes

The error with respect to the exact solution $p$ is measured using the following two quantities:

$$
\operatorname{Err}_{2, \mathcal{V}}:=\frac{\left\|\mathrm{p}-\mathrm{R}_{\mathcal{V}}(p)\right\|_{2, \mathcal{V}}}{\left\|\mathrm{R}_{\mathcal{V}}(p)\right\|_{2, \mathcal{V}}}, \quad \operatorname{Err}_{\mathrm{ad}, \mathcal{V}}:=\frac{\left\|\mathrm{p}-\mathrm{R}_{\mathcal{V}}(p)\right\|_{\mathrm{ad}, \mathcal{V}}}{\left\|\mathrm{R}_{\mathcal{V}}(p)\right\|_{\mathrm{ad}, \mathcal{V}}}
$$

In our numerical tests, the integrals for the source term and the boundary data are computed using a fourth-order quadrature on elementary sub-simplices of each polyhedral cell.

\subsection{Anisotropic diffusion and variable advection velocity}

We consider the conservative form of the scheme (4.19), where the bilinear form $\mathrm{A}_{\beta}$ is given by (3.8). The exact solution is $p(x, y, z)=1+\sin (\pi x) \sin \left(\pi\left(y+\frac{1}{2}\right)\right) \sin \left(\pi\left(z+\frac{1}{3}\right)\right)$, and the diffusive tensor $\boldsymbol{\lambda}$ and the velocity field $\boldsymbol{\beta}$ are equal to (in the canonical basis of $\mathbb{R}^{3}$ )

$$
\boldsymbol{\lambda}=\left(\begin{array}{ccc}
1 & 0.5 & 0 \\
0.5 & 1 & 0.5 \\
0 & 0.5 & 1
\end{array}\right), \quad \boldsymbol{\beta}=\left(\begin{array}{c}
y-1 / 2 \\
1 / 2-x \\
z
\end{array}\right)
$$

so that the velocity field satisfies hypothesis $(\boldsymbol{\beta 1})$ for the conservative form (see Remark 3.2). We consider the discrete contraction operator $I_{\beta}^{\sim \widetilde{\mathcal{F}}}$ built using full upwinding as in Section 3.2 and Péclet-based upwinding as in Section 4.3 using the Sharfetter-Gummel map.

Figure 6 presents the numerical results, which reflect the theoretical analysis with convergence rates between one and two. The use of Péclet-based upwinding leads to lower errors than full upwinding; the improvement is more pronounced on the SkP mesh sequence than on the other sequences, and is observed on the finer meshes. 

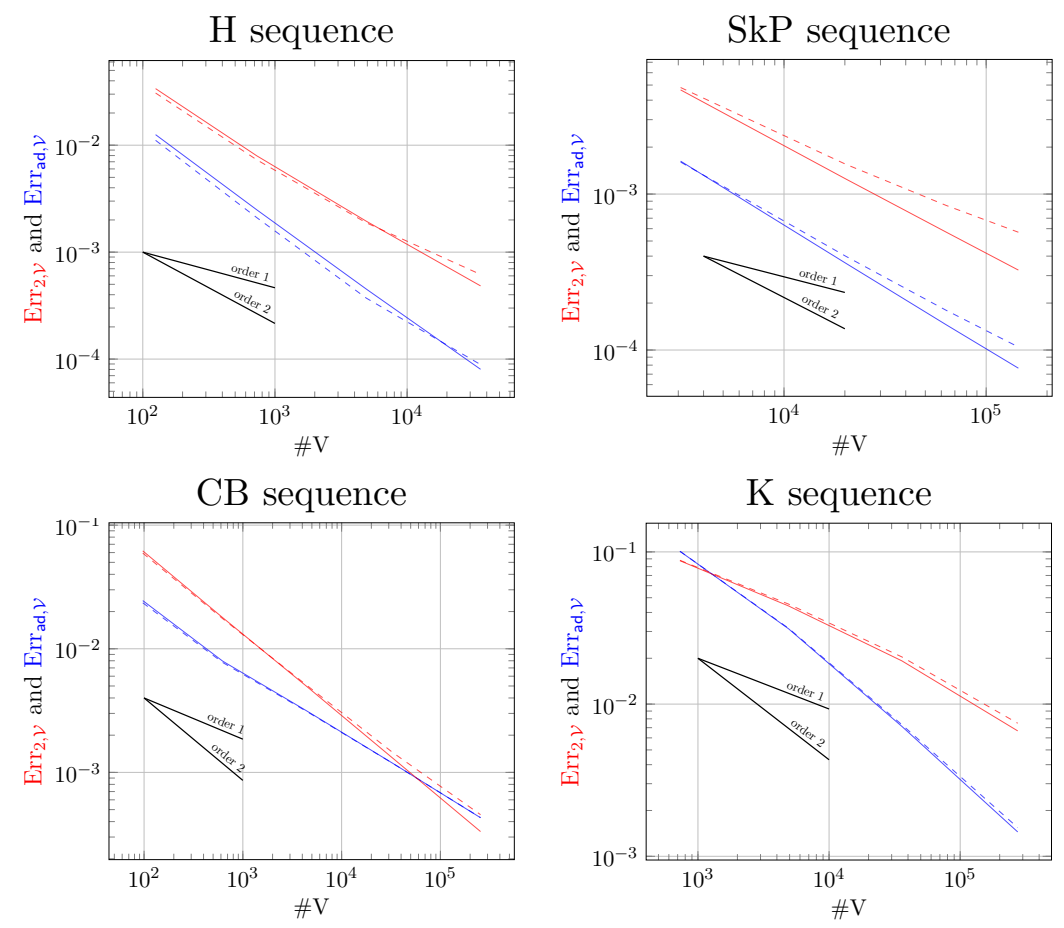

Figure 6: Test case 6.1: Convergence curves for the two error measures on the four mesh families using full upwinding (dashed lines) or Péclet-based upwinding (solid lines); first- and second-order slopes are indicated.

\subsection{Exponential boundary layer with constant advection velocity}

The second test case investigates the behavior of the CDO scheme in the presence of an exponential boundary layer resulting from small diffusion. We consider an isotropic diffusive tensor, i.e. $\boldsymbol{\lambda}=\lambda \mathbf{I d}$, and a constant vector field $\boldsymbol{\beta}$ with components $(2,3,0)$, so that assumption $(\boldsymbol{\beta 2})$ is satisfied. The exact solution is $p(x, y, z)=$ $\left(x-e^{\frac{2(x-1)}{\lambda}}\right)\left(y^{2}-e^{\frac{3(y-1)}{\lambda}}\right)$ and exhibits a boundary layer near $x=1$ and $y=1$ when $\lambda$ tends to 0 .

Figure 7 reports numerical results for $\lambda=1$ (solid lines) and $\lambda=10^{-4}$ (dashed lines). Note that in this second case, the considered meshes do not resolve the boundary layer. The transition between the two convergence regimes as predicted by Theorem 4.7 is clearly visible. The present test case is also considered by Da Veiga et al. [5] on the same SkP mesh sequence with a different scheme, where similar convergence rates are reported but with somewhat larger error values.

\section{Analysis of CDO schemes for pure diffusion}

\subsection{Proof of Lemma 4.2}

Property $(\mathbf{N} 1)$ implies that, for all $\left(\mathbf{q}^{\partial}, \mathrm{g}\right) \in \mathcal{V}^{\partial} \times \mathcal{E}$,

$$
\begin{aligned}
\llbracket \mathrm{q}^{\partial}, \mathrm{N}_{\boldsymbol{\lambda}}^{\partial}(\mathrm{g}) \rrbracket_{(\nu \tilde{\mathcal{F}})^{\partial}} & =\sum_{f \in \mathrm{F}^{\partial}} \llbracket \mathrm{q}_{f}^{\partial}, \mathrm{N}_{\boldsymbol{\lambda}}^{\partial f}\left(\mathrm{~g}_{c}\right) \rrbracket_{(\nu \tilde{\mathcal{F}})_{f}^{\partial}}=\sum_{f \in \mathrm{F}^{\partial}} \sum_{v \in \mathrm{V}_{f}^{\partial}} \mathbf{q}_{f, v}^{\partial}\left(\mathrm{N}_{\lambda}^{\partial f}\left(\mathrm{~g}_{c}\right)\right)_{\tilde{f}^{\partial}(v)} \\
& \leq c_{\mathrm{N}} \sum_{f \in \mathrm{F}^{\partial}}\left\|\mathbf{q}_{f}^{\partial}\right\|_{\lambda / h, f}\left\|\mathrm{~g}_{c}\right\|_{\boldsymbol{\lambda}, c} \leq c_{\mathrm{N}}\left\|\mathbf{q}^{\partial}\right\|_{\lambda / h}\|\mathrm{~g}\|_{\boldsymbol{\lambda}},
\end{aligned}
$$

where we have used the local assembly of $\mathrm{N}_{\lambda}^{\partial}$ on the first line (with $c=c(f)$ ), the discrete Cauchy-Schwarz inequalities for the summations $\sum_{v \in \mathrm{V}_{f}^{\partial}}$ and $\sum_{f \in \mathrm{F}^{\partial}}$, and the fact that $\sum_{f \in \mathrm{F}^{\partial}}\left\|\mathrm{g}_{c}\right\|_{\boldsymbol{\lambda}, c}^{2} \leq\|\mathrm{g}\|_{\boldsymbol{\lambda}}^{2}$ on the second 

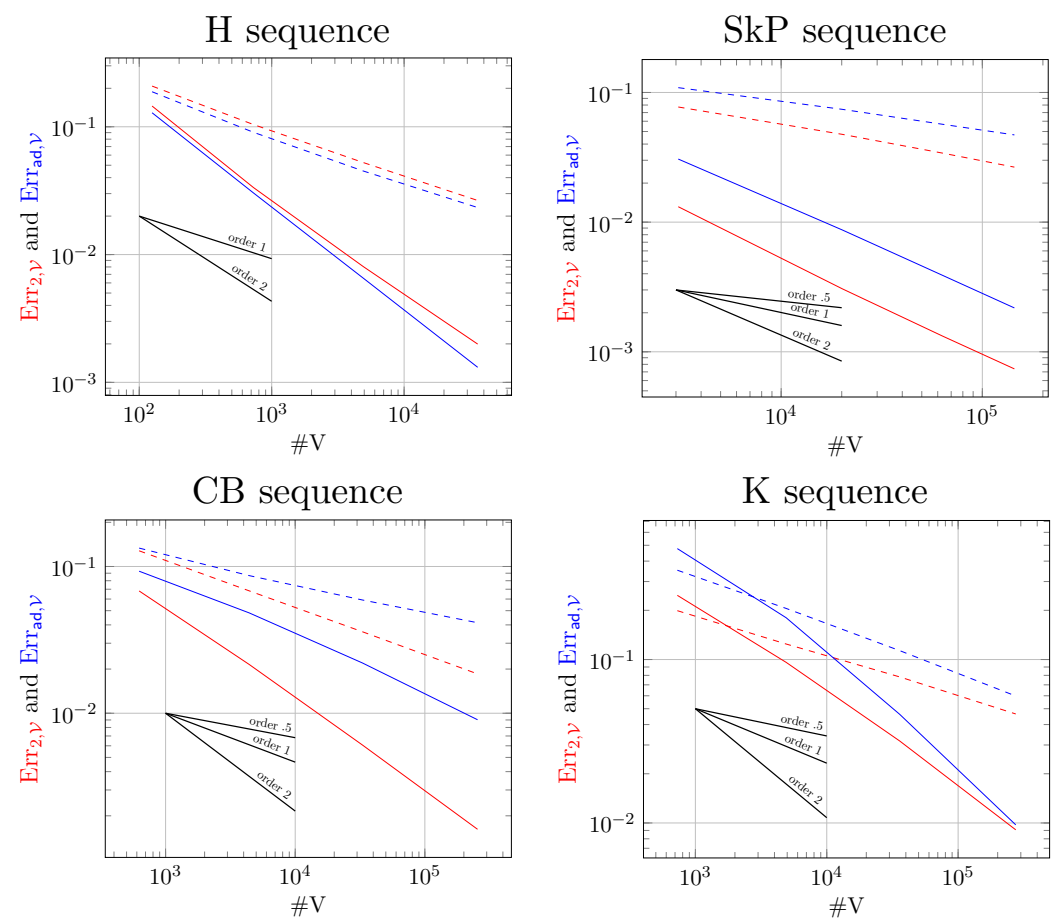

Figure 7: Test case 6.2: Convergence curves for the two error measures on the four mesh families using Péclet-based upwinding for $\lambda=1$ (solid lines) and $\lambda=10^{-4}$ (dashed lines); half-, first-, and second-order slopes are indicated.

line. As a result, we infer that

$$
\mathrm{A}_{\boldsymbol{\lambda}}(\mathrm{q}, \mathrm{q}) \geq\|\operatorname{GRAD}(\mathrm{q})\|_{\lambda}^{2}-c_{\mathrm{N}}\|\operatorname{GRAD}(\mathrm{q})\|_{\lambda}\|\mathrm{q}\|_{\lambda / h}+\eta_{0}\|\mathrm{q}\|_{\lambda / h}^{2}
$$

To conclude, we use the quadratic inequality $x^{2}-2 \gamma x y+\delta y^{2} \geq \frac{\delta-\gamma^{2}}{1+\delta}\left(x^{2}+y^{2}\right)$ (valid for any real numbers $x, y, \gamma, \delta$ with $\delta \geq 0)$ with $\gamma=\frac{1}{2} c_{\mathrm{N}}$ and $\delta=\eta_{0}$ and observe that the choice $\eta_{0} \geq 1+\frac{1}{2} c_{\mathrm{N}}^{2}$ implies $\delta \geq 1+2 \gamma^{2}$ so that $\frac{\delta-\gamma^{2}}{1+\delta} \geq \frac{1}{2}$. Finally, the well-posedness of (4.3) follows from (4.14).

\subsection{Proof of Lemma 4.3}

Owing to Lemma 4.2, it suffices to show that $\mathrm{A}_{\boldsymbol{\lambda}}\left(\mathrm{p}-\mathrm{R}_{\mathcal{V}}(p), \mathbf{q}\right)=\mathrm{E}_{\boldsymbol{\lambda}}(p, \mathbf{q})$. To prove this, we observe that

$$
\llbracket \mathrm{q}, \mathrm{s} \rrbracket_{\mathcal{V} \tilde{\mathcal{C}}}=-\llbracket \mathrm{q}, \mathrm{R}_{\widetilde{\mathcal{C}}}(\nabla \cdot(\boldsymbol{\lambda} \nabla p)) \rrbracket_{\mathcal{V} \tilde{\mathcal{C}}}=-\llbracket \mathrm{q}, \widetilde{\operatorname{DIV}}\left(\mathrm{R}_{\widetilde{\mathcal{F}}}(\boldsymbol{\lambda} \nabla p)\right) \rrbracket_{\mathcal{V} \tilde{\mathcal{C}}}-\llbracket \mathrm{q}^{\partial}, \mathrm{R}_{\widetilde{\mathcal{F}} \partial}(\boldsymbol{n} \cdot \boldsymbol{\lambda} \cdot \nabla p) \rrbracket_{(\mathcal{\nu} \tilde{\mathcal{F}})},
$$

owing to $(2.2 \mathrm{~b})$, and we use (4.3) and (4.4) to conclude, as well as $\mathrm{R}_{\mathcal{V}^{\partial}}\left(p_{\mathrm{D}}\right)=\left(\mathrm{R}_{\mathcal{V}}(p)\right)^{\partial}$.

\subsection{Proof of Theorem 4.4}

Let $T_{1}, T_{2}$ be the two terms in the right-hand side of (4.17). Recall that $\operatorname{GRAD}\left(\operatorname{R}_{\mathcal{V}}(p)\right)=\operatorname{R}_{\mathcal{E}}(\nabla p)$. The term $T_{1}$ has already been bounded in [9]; we present here a somewhat simpler proof avoiding the algebraic identity on the inverse of the discrete Hodge operator. Let $\boldsymbol{G}_{c}$ denote the mean-value of $\nabla p$ in $c$. Owing to 
the local assembly (4.5) and to (H2), we infer that

$$
\begin{aligned}
T_{1} & =\sum_{c \in \mathrm{C}} \llbracket \operatorname{GRAD}_{c}(\mathbf{q}), \mathrm{R}_{\widetilde{\mathcal{F}}_{c}}(\boldsymbol{\lambda} \cdot \nabla p)-\mathrm{H}_{\lambda}^{(\mathcal{E} \tilde{\mathcal{F}})_{c}}\left(\mathrm{R}_{\mathcal{E}_{c}}(\nabla p) \rrbracket_{(\tilde{\mathcal{F}})_{c}}\right. \\
& =\sum_{c \in \mathrm{C}} \llbracket \operatorname{GRAD}_{c}(\mathbf{q}), \mathrm{R}_{\widetilde{\mathcal{F}}_{c}}\left(\boldsymbol{\lambda} \cdot\left(\nabla p-\boldsymbol{G}_{c}\right)\right) \rrbracket_{(\tilde{\mathcal{F}} \tilde{\mathcal{F}})_{c}}-\sum_{c \in \mathrm{C}} \llbracket \operatorname{GRAD}_{c}(\mathbf{q}), \mathrm{H}_{\boldsymbol{\lambda}}^{(\mathcal{\varepsilon})_{c}}\left(\mathrm{R}_{\mathcal{E}_{c}}\left(\nabla p-\boldsymbol{G}_{c}\right)\right) \rrbracket_{(\mathcal{E} \tilde{\mathcal{F}})_{c}},
\end{aligned}
$$

and we denote by $T_{1,1}, T_{1,2}$ the two terms in the right-hand side. The Cauchy-Schwarz inequality, mesh regularity, and the lower bound in (H1) imply that

$$
\begin{aligned}
\left|T_{1,1}\right| & \lesssim\|\operatorname{GRAD}(\mathbf{q})\|_{\lambda}\left(\sum_{c \in \mathrm{C}} \sum_{e \in \mathrm{E}_{c}} \rho_{c} \lambda_{\sharp, c} h_{c}^{-1}\left\|\nabla p-\boldsymbol{G}_{c}\right\|_{L^{1}\left(\tilde{f}_{c}(e)\right)}^{2}\right)^{\frac{1}{2}} \\
& \lesssim\|\operatorname{GRAD}(\mathbf{q})\|_{\lambda}\left(\sum_{c \in \mathrm{C}} \rho_{c} \lambda_{\sharp, c} h_{c}^{2}|p|_{H^{2}(c)}^{2}\right)^{\frac{1}{2}} \cdot
\end{aligned}
$$

Similarly, the Cauchy-Schwarz inequality for $\mathrm{H}_{\lambda}^{(\tilde{\mathcal{F}})_{c}}\left(\right.$ i.e., $\llbracket g_{1}, \mathrm{H}_{\lambda}^{(\mathcal{\mathcal { F }})_{c}}\left(\mathrm{~g}_{2}\right) \rrbracket_{(\mathcal{E} \tilde{\mathcal{F}})_{c}} \leq\left\|g_{1}\right\|_{\lambda, c}\left\|g_{2}\right\|_{\lambda, c}$ for all $\mathrm{g}_{1}, \mathrm{~g}_{2} \in$ $\left.\mathcal{E}_{c}\right)$ and the upper bound in (H1) imply that

$$
\left|T_{1,2}\right| \lesssim\|\operatorname{GRAD}(\mathbf{q})\|_{\lambda}\left(\sum_{c \in \mathrm{C}} \sum_{e \in \mathrm{E}_{c}} \lambda_{\sharp, c} h_{c}\left\|\nabla p-\boldsymbol{G}_{c}\right\|_{L^{1}(e)}^{2}\right)^{\frac{1}{2}} \lesssim\|\operatorname{GRAD}(\mathbf{q})\|_{\boldsymbol{\lambda}}\left(\sum_{c \in \mathrm{C}} \lambda_{\sharp, c} h_{c}^{2}|p|_{H^{2}(c)}^{2}\right)^{\frac{1}{2}} .
$$

Turning to $T_{2}$, we use the local assembly (4.6) and (N2) to infer that, with $c=c(f)$,

$$
\begin{aligned}
T_{2} & =\sum_{f \in \mathrm{F}^{\partial}} \llbracket \mathrm{q}_{f}^{\partial}, \mathrm{R}_{\widetilde{\mathcal{F}}_{f}^{\partial}}(\boldsymbol{n} \cdot \boldsymbol{\lambda} \cdot \nabla p)-\mathrm{N}_{\boldsymbol{\lambda}}^{\partial f}\left(\mathrm{R}_{\mathcal{E}_{c}}(\nabla p)\right) \rrbracket_{(\mathcal{\nu} \tilde{\mathcal{F}})_{f}^{\partial}} \\
& =\sum_{f \in \mathrm{F}^{\partial}} \llbracket \mathrm{q}_{f}^{\partial}, \mathrm{R}_{\widetilde{\mathcal{F}}_{f}^{\partial}}\left(\boldsymbol{n} \cdot \boldsymbol{\lambda} \cdot\left(\nabla p-\boldsymbol{G}_{c}\right)\right) \rrbracket_{\left(\mathcal{\nu} \tilde{\mathcal{F}}_{f}^{\partial}\right.}-\sum_{f \in \mathrm{F}^{\partial}} \llbracket \mathrm{q}_{f}^{\partial}, \mathrm{N}_{\boldsymbol{\lambda}}^{\partial f}\left(\mathrm{R}_{\mathcal{E}_{c}}\left(\nabla p-\boldsymbol{G}_{c}\right)\right) \rrbracket_{(\mathcal{\nu} \tilde{\mathcal{F}})_{f}^{\partial}},
\end{aligned}
$$

and we denote by $T_{2,1}$ and $T_{2,2}$ the two terms in the right-hand side. The Cauchy-Schwarz inequality implies that

$$
\begin{aligned}
\left|T_{2,1}\right| & \leq\left\|\mathbf{q}^{\partial}\right\|_{\lambda / h}\left(\sum_{f \in \mathrm{F}^{\partial}} \sum_{v \in \mathrm{V}_{f}^{\partial}} \lambda_{\sharp, c} h_{c}^{-1}\left\|\nabla p-\boldsymbol{G}_{c}\right\|_{L^{1}\left(\tilde{f}_{f}^{\partial}(v)\right)}^{2}\right)^{\frac{1}{2}} \\
& \lesssim\left\|\mathbf{q}^{\partial}\right\|_{\lambda / h}\left(\sum_{f \in \mathrm{F}^{\partial}} \lambda_{\sharp, c} h_{c}^{2}|p|_{H^{2}(c)}^{2}\right)^{\frac{1}{2}},
\end{aligned}
$$

while using (N1) and proceeding as above, we infer a similar bound on $T_{2,2}$. The proof is complete since $\rho_{c} \geq 1$ by definition.

Acknowledgement. The authors thank Jérôme Bonelle (EDF R\&D) for fruitful discussions. 


\section{References}

[1] P. Angot, V. Dolejší, M. Feistauer, and J. Felcman. Analysis of a combined barycentric finite volume-nonconforming finite element method for nonlinear convection-diffusion problems. Appl. Math., 43(4):263-310, 1998.

[2] B. Ayuso and L. D. Marini. Discontinuous Galerkin methods for advection-diffusion-reaction problems. SIAM J. Numer. Anal., 47(2):1391-1420, 2009.

[3] K. Baba and M. Tabata. On a conservative upwind finite element scheme for convective diffusion equations. RAIRO Anal. Numér., 15(1):3-25, 1981.

[4] L. Beirão da Veiga, F. Brezzi, A. Cangiani, G. Manzini, L. D. Marini, and A. Russo. Basic principles of virtual element methods. M3AS Math. Models Methods Appl. Sci., 199(23):199-214, 2013.

[5] L. Beirão da Veiga, J. Droniou, and G. Manzini. A unified approach for handling convection terms in finite volumes and mimetic discretization methods for elliptic problems. IMA J. Numer. Anal., 31(4):1357-1401, 2011.

[6] P. Bochev, M. Perego, and K. Peterson. Formulation and analysis of a parameter-free stabilized finite element method. SIAM J. Num. Anal., 53:2363-2388, 2015.

[7] J. Bonelle. Compatible Discrete Operator schemes on polyhedral meshes for elliptic and Stokes equations. $\mathrm{PhD}$ thesis, Université Paris Est, 2014.

[8] J. Bonelle, D. A. Di Pietro, and A. Ern. Low-order reconstruction operators on polyhedral meshes: Application to Compatible Discrete Operator schemes. Cumput. Aided Geom. Design, 35/36:27-41, 2015.

[9] J. Bonelle and A. Ern. Analysis of compatible discrete operator schemes for elliptic problems on polyhedral meshes. ESAIM Math. Model. Numer. Anal., 48(2):553-581, 2014.

[10] J. Bonelle and A. Ern. Analysis of compatible discrete operator schemes for stokes problems on polyhedral meshes. IMA J. Numer. Anal., 34(4):553-581, 2014.

[11] A. Bossavit. Computational electromagnetism. Electromagnetism. Academic Press, Inc., San Diego, CA, 1998. Variational formulations, complementarity, edge elements.

[12] A. Bossavit. Computational electromagnetism and geometry. J. Japan Soc. Appl. Electromagn. \& Mech., 7-8:150-9 (no 1), 294-301 (no 2), 401-8 (no 3), 102-9 (no 4), 203-9 (no 5), 372-7 (no 6), 1999-2000.

[13] A. Bossavit. Extrusion, contraction: their discretization via Whitney forms. COMPEL, 22(3):470-480, 2003. Selected papers from the 10th International IGTE Symposium on Numerical Field Computation (Graz, 2002).

[14] F. Brezzi, L. D. Marini, and E. Süli. Discontinuous Galerkin methods for first-order hyperbolic problems. Math. Models Methods Appl. Sci., 14(12):1893-1903, 2004.

[15] S. H. Christiansen. A construction of spaces of compatible differential forms on cellular complexes. Math. Models Methods Appl. Sci., 18(5):739-757, 2008. 
[16] L. Codecasa, R. Specogna, and F. Trevisan. A new set of basis functions for the discrete geometric approach. J. Comput. Phys., 229(19):7401-7410, 2010.

[17] P. Deuring, R. Eymard, and M. Mildner. L L ${ }^{2}$-stability independent of diffusion for a Finite ElementFinite Volume discretization of a linear convection-diffusion equation. SIAM J. Numer. Anal., 53:508$526,2015$.

[18] A. Devinatz, R. Ellis, and A. Friedman. The asymptotic behavior of the first real eigenvalue of second order elliptic operators with a small parameter in the highest derivatives. II. Indiana Univ. Math. J., 23:991-1011, 1973-1974.

[19] D. A. Di Pietro, J. Droniou, and A. Ern. A discontinuous-skeletal method for advection-diffusionreaction on general meshes. SIAM J. Numer. Anal, 53:2135-2157, 2015.

[20] D. A. Di Pietro and A. Ern. Mathematical aspects of discontinuous Galerkin methods, volume 69 of Mathématiques \& Applications (Berlin) [Mathematics \& Applications]. Springer, Heidelberg, 2012.

[21] A. Ern and J.-L. Guermond. Discontinuous Galerkin methods for Friedrichs' systems. I. General theory. SIAM J. Numer. Anal., 44(2):753-778, 2006.

[22] A. Ern and J.-L. Guermond. Discontinuous Galerkin methods for Friedrichs' systems. II. Second-order elliptic PDEs. SIAM J. Numer. Anal., 44(6):2363-2388, 2006.

[23] A. Ern, J.-L. Guermond, and G. Caplain. An intrinsic criterion for the bijectivity of Hilbert operators related to Friedrichs' systems. Comm. Partial Differential Equations, 32(1-3):317-341, 2007.

[24] R. Eymard, G. Henry, R. Herbin, F. Hubert, R. Klofkorn, and G. Manzini. 3D benchmark on discretization schemes for anisotropic diffusion problems on general grids. In Proceedings of Finite Volumes for Complex Applications VI, pages 895-930. Springer, 2011.

[25] H. Heumann and R. Hiptmair. Extrusion contraction upwind schemes for convection-diffusion problems. Technical Report 2008-30, ETH Zürich, October 2008.

[26] H. Heumann and R. Hiptmair. Eulerian and semi-Lagrangian methods for convection-diffusion for differential forms. Discrete Contin. Dyn. Syst., 29(4):1471-1495, 2011.

[27] D. Hilhorst and M. Vohralík. A posteriori error estimates for combined finite volume-finite element discretizations of reactive transport equations on nonmatching grids. Comput. Methods Appl. Mech. Engrg., 200(5-8):597-613, 2011.

[28] R. Hiptmair. Discrete Hodge operators. Numer. Math., 90(2):265-289, 2001.

[29] R. Hiptmair. Finite elements in computational electromagnetism. Acta Numer., 11:237-339, 2002.

[30] C. Johnson and J. Pitkäranta. An analysis of the discontinuous Galerkin method for a scalar hyperbolic equation. Math. Comp., 46(173):1-26, 1986.

[31] P. Mullen, A. McKenzie, D. Pavlov, L. Durant, Y. Tong, E. Kanso, J. E. Marsden, and M. Desbrun. Discrete Lie advection of differential forms. Found. Comput. Math., 11(2):131-149, 2011. 
[32] J. Nitsche. Über ein Variationsprinzip zur Lösung von Dirichlet-Problemen bei Verwendung von Teilräumen, die keinen Randbedingungen unterworfen sind. Abh. Math. Sem. Univ. Hamburg, 36:9-15, 1971. Collection of articles dedicated to Lothar Collatz on his sixtieth birthday.

[33] K. Ohmori and T. Ushijima. A technique of upstream type applied to a linear nonconforming finite element approximation of convective diffusion equations. RAIRO Anal. Numér., 18(3):309-332, 1984.

[34] A. Palha, P. Pinto Rebelo, and M. Gerritsma. Mimetic spectral element advection. arXiv:1304.6926, 2013.

[35] H.-G. Roos, M. Stynes, and L. Tobiska. Robust numerical methods for singularly perturbed differential equations, volume 24 of Springer Series in Computational Mathematics. Springer-Verlag, Berlin, second edition, 2008. Convection-diffusion-reaction and flow problems.

[36] T. Tarhasaari, L. Kettunen, and A. Bossavit. Some realizations of a discrete hodge operator: A reinterpretation of finite element techniques. IEEE Transactions on magnetics, 35(3):1494-1497, may 1999.

[37] E. Tonti. The mathematical structure of classical and relativistic physics. Modeling and Simulation in Science, Engineering and Technology. Birkhäuser/Springer, New York, 2013. A general classification diagram. 